\title{
ON REGULAR SOLUTIONS TO TWO-DIMENSIONAL THERMOVISCOELASTICITY
}

Abstract. A two-dimensional thermoviscoelastic system of Kelvin-Voigt type with strong dependence on temperature is considered. The existence and uniqueness of a global regular solution is proved without small data assumptions. The global existence is proved in two steps. First, a global a priori estimate is derived by applying anisotropic Sobolev spaces with a mixed norm. Then local existence, proved by the method of successive approximations for a sufficiently small time interval, is extended step by step in time. By a two-dimensional solution we mean that all the relevant quantities depend on two space variables only.

1. Introduction. This article is devoted to the problem of global existence and uniqueness of regular solutions to a two-dimensional (2d) thermoviscoelasticity system for small strains which is still strongly nonlinear. The system describes homogeneous isotropic linearly-responding viscoelastic materials in the Kelvin-Voigt rheology at small strains. We assume that the specific heat and the elasticity tensor depend on the temperature in a very special way.

The paper is a companion paper to [GZ4]. Compared to [GZ4], the proof of the global a priori estimate is only sketched (see Section 5) but the proof of local existence is added.

Recently in [PZ1] global existence of regular solutions to three-dimensional thermoviscoelasticity with specific heat linearly increasing with temperature and with constant heat conductivity has been proved. This setting

2010 Mathematics Subject Classification: Primary 74B20, 35K50; Secondary 35Q72, $74 \mathrm{~F} 05$.

Key words and phrases: thermoviscoelastic system, Kelvin-Voigt type materials, anisotropic Sobolev spaces with a mixed norm, a priori estimates, global existence.

Received 21 March 2016; revised 15 June 2016.

Published online 23 September 2016. 
is a particular case of systems considered in [BG, R1]. Existence of weak solutions for generalized thermoviscoelastic materials with various kinds of boundary conditions has been proved in [R2, $[\mathrm{RR}]$. Moreover, the papers of Roubíček [R1, R2, R3] and Rossi-Roubíček [RR] present a deep physical background on thermoviscoelastic materials.

Pioneering papers on global regular solutions to one-dimensional thermoviscoelasticity are $[\mathrm{SL}, \mathrm{D}, \mathrm{DH}$, and the spherical case is considered in $[\mathrm{GA}$. Recently, global existence of large solutions to spherically symmetric nonlinear viscoelasticity has been proved in [GZ1, GZ2].

In this paper we consider a two-dimensional thermoviscoelastic system with the temperature dependent specific heat of the form $c_{*}=c_{v} \theta^{\sigma}$ with $1 / 2<\sigma<1, c_{v}$ a positive constant, and with constant heat conductivity. This setting is a particular case of systems addressed in [R1]. Moreover, the stress tensor is given by a linear thermoviscoelastic law of Kelvin-Voigt type (cf. [EJK, Chapter 5.4]). The aim of this paper is to prove existence of global regular solutions to the $2 \mathrm{~d}$-thermoviscoelastic system without smallness assumptions on data and for $\sigma$ as small as possible. In the 3d-case (see [PZ1]) existence of global regular solutions for large data is only proved for $\sigma=1$. Restricting our considerations to the $2 \mathrm{~d}$ case we are able to use the specific heat $c_{*}=c_{v} \theta^{\sigma}, \sigma \in(1 / 2,1)$.

The proof of global existence is in two main steps. First we need a global a priori estimate in Sobolev spaces $W_{p, p_{0}}^{2,1}\left(\Omega^{T}\right)$ with mixed norm. This is possible because equations for displacement and temperature are parabolic. This idea was developed in [PZ1. Since $c_{*}=c_{v} \theta^{\sigma}$ is the coefficient heat near $\theta_{, t}$ we need continuity of $\theta$ to apply the theory for parabolic equations, so $p$ and $p_{0}$ must be sufficiently large. Next, we prove local existence in $W_{p, p_{0}}^{2,1}\left(\Omega^{T}\right)$-spaces by the method of successive approximations. Combining these two steps we prove the main result: global existence of regular solutions with large data.

Thus we consider the following thermoviscoelasticity system:

$$
\begin{array}{ll}
u_{, t t}-\operatorname{div}\left[\left(A_{1} \varepsilon_{, t}\right)+\left(A_{2} \varepsilon\right)+A \theta\right]=b & \text { in } \Omega^{T}=\Omega \times(0, T), \\
c_{v} \theta^{\sigma} \theta_{, t}-\varkappa \Delta \theta=\theta A \varepsilon_{, t}+\left(A_{1} \varepsilon_{, t}\right) \cdot \varepsilon_{, t}+g & \text { in } \Omega^{T},
\end{array}
$$

where $\Omega \subset \mathbb{R}^{2}$, with boundary $S$, is bounded, and $\sigma$ is a positive constant. We add the boundary conditions

$$
u=0, \quad \bar{n} \cdot \nabla \theta=0 \quad \text { on } S^{T}=S \times(0, T),
$$

where $\bar{n}$ is the unit outward normal vector to $S$, and the initial conditions

$$
\left.u\right|_{t=0}=u_{0},\left.\quad u_{, t}\right|_{t=0}=u_{1},\left.\quad \theta\right|_{t=0}=\theta_{0} \quad \text { in } \Omega .
$$

The field $u: \Omega^{T} \rightarrow \mathbb{R}^{2}$ is the displacement, and $\theta: \Omega^{T} \rightarrow \mathbb{R}_{+}$is the absolute temperature. The second order tensors $\varepsilon=\left\{\varepsilon_{i j}\right\}_{i, j=1,2}$ and $\varepsilon_{, t}=\left\{\varepsilon_{i j, t}\right\}_{i, j=1,2}$ 
denote, respectively, the fields of the linearized strain and the strain rate, which are defined by

$$
\varepsilon=\varepsilon(u)=\frac{1}{2}\left(\nabla u+(\nabla u)^{\mathrm{T}}\right), \quad \varepsilon_{, t}=\varepsilon\left(u_{, t}\right)=\frac{1}{2}\left(\nabla u_{, t}+\left(\nabla u_{, t}\right)^{\mathrm{T}}\right) .
$$

Equation 1.1 is the linear momentum balance with the stress tensor given by a linear thermoviscoelastic law of Kelvin-Voigt type (cf. EJK. Chapter $5.4])$

$$
\mathbb{S}=A_{1} \varepsilon_{, t}+A_{2} \varepsilon+A \theta .
$$

The fourth order tensors $A_{1}=\left\{A_{1 i j k l}\right\}_{i, j, k, l=1,2}$ and $A_{2}=\left\{A_{2 i j k l}\right\}_{i, j, k, l=1,2}$ are respectively, the linear viscoelasticity and the elasticity tensors, defined by the Hook law

$$
\varepsilon \mapsto A_{i} \varepsilon=\lambda_{i} \operatorname{tr} \varepsilon \mathbb{I}+2 \mu_{i} \varepsilon, \quad i=1,2,
$$

where $\lambda_{1}, \mu_{1}$ are the viscosity constants and $\lambda_{2}, \mu_{2}$ are the Lamé constants, both $\lambda_{1}, \mu_{1}$ and $\lambda_{2}, \mu_{2}$ with values within the elasticity range

$$
\mu_{i}>0, \quad 3 \lambda_{i}+2 \mu_{i}>0, \quad i=1,2,
$$

and $\mathbb{I}$ is the unit matrix. In the case of 1.1 , 1.2 the free energy is specified by

$$
f(\varepsilon, \theta)=f_{*}(\theta)+W(\varepsilon, \theta),
$$

where

$$
f_{*}(\theta)=-\frac{c_{v}}{\sigma(\sigma+1)} \theta^{\sigma+1}, \quad c_{v}=\text { const }>0,
$$

is the caloric energy, and

$$
\begin{aligned}
W(\varepsilon, \theta) & =\frac{1}{2}(\varepsilon-\theta \alpha) \cdot A_{2}(\varepsilon-\theta \alpha)-\frac{1}{2} \theta^{2} \alpha \cdot\left(A_{2} \alpha\right) \\
& =\frac{1}{2} \varepsilon \cdot\left(A_{2} \varepsilon\right)-\theta \varepsilon \cdot\left(A_{2} \alpha\right)
\end{aligned}
$$

is the elastic energy, where $\alpha=\left(\alpha_{i j}\right)_{i, j=1,2}$, with constant $\alpha_{i j}$, is the symmetric thermal expansion tensor. For notational simplicity we introduce the second order symmetric tensor $A$ given by

$$
-A_{2} \alpha=A \text {. }
$$

From 1.10 the specific heat takes the form

$$
c_{*}=-\theta f_{*}^{\prime \prime}(\theta)=c_{v} \theta^{\sigma} .
$$

The dissipation potential corresponding to system (1.1), 1.2 is given by

$$
\mathcal{D}=\frac{1}{2 \theta} \varepsilon_{, t} \cdot\left(A_{1} \varepsilon_{, t}\right)+\frac{k}{2} \theta^{2}\left|\nabla \frac{1}{\theta}\right|^{2},
$$

where $k>0$ is the constant heat conductivity. Hence, by the Fourier law the heat flux takes the form

$$
q=k \theta^{2} \nabla \frac{1}{\theta}=-k \nabla \theta
$$


The dot will denote the scalar product in $\mathbb{R}^{2}$ or in $\mathbb{R}^{2} \times \mathbb{R}^{2}$. Let $u=\left\{u_{i}\right\}$, $v=\left\{v_{i}\right\}, S=\left\{S_{i j}\right\}, R=\left\{R_{i j}\right\}, A_{m}=\left\{A_{m i j k l}\right\}, m=1,2, \varepsilon=\left\{\varepsilon_{i j}\right\}, A=$ $\left\{A_{i j}\right\}$. Then we apply the summation convention: $u \cdot v=u_{i} v_{i}, S \cdot R=S_{i j} R_{i j}$, $(S u)_{i}=S_{i j} u_{j},\left(A_{m} \varepsilon\right)_{i j}=A_{m i j k l} \varepsilon_{k l},\left(A_{m} \varepsilon\right) \cdot \varepsilon=A_{m i j k l} \varepsilon_{k l} \varepsilon_{i j}$. Moreover, we have

$$
\nabla \cdot\left(A_{m} \varepsilon\right)_{i}=\partial_{x_{j}}\left(A_{m i j k l} \varepsilon_{k l}\right) .
$$

We define linear viscosity and elasticity tensors $Q_{1}$ and $Q_{2}$ by

$$
u \mapsto Q_{i} u=\nabla \cdot\left(A_{i} \varepsilon(u)\right)=\mu_{i} \Delta u+\left(\lambda_{i}+\mu_{i}\right) \nabla \nabla \cdot u, \quad i=1,2 .
$$

In view of (1.16) equation 1.1) takes the form

$$
u_{, t t}-Q_{1} u_{, t}-Q_{2} u=\nabla \cdot(A \theta)+b .
$$

We assume that the tensors $A_{m}$ satisfy the symmetry conditions

$$
\left(A_{m}\right)_{i j k l}=\left(A_{m}\right)_{j i k l}=\left(A_{m}\right)_{k l i j}, \quad m=1,2,
$$

and coercivity and boundedness

$$
a_{m *}|\varepsilon|^{2} \leq\left(A_{m} \varepsilon\right) \cdot \varepsilon \leq a_{m}^{*}|\varepsilon|^{2}, \quad m=1,2,
$$

where

$$
a_{m *}=\min \left\{3 \lambda_{m}+2 \mu_{m}, 2 \mu_{m}\right\}, \quad a_{m}^{*}=\max \left\{3 \lambda_{m}+2 \mu_{m}, 2 \mu_{m}\right\} .
$$

Let us consider the problem

$$
\begin{array}{ll}
Q_{m} u=f_{m} & \text { in } \Omega \subset \mathbb{R}^{2}, m=1,2, \\
u=0 & \text { on } S .
\end{array}
$$

Lemma 1.1 ([LM, Ch. 2]). Let $f_{m} \in L_{2}(\Omega)$ and suppose conditions (1.18, 1.19 hold. Then there exists a unique solution to 1.20 such that $u \in H^{2}(\Omega)$ and

$$
\|u\|_{H^{2}(\Omega)} \leq c_{m}\left\|f_{m}\right\|_{L_{2}(\Omega)}, \quad m=1,2 .
$$

For $u \in \operatorname{Dom} Q_{m} \cap H_{0}^{1}(\Omega)$ inequality 1.21 can be written in the form

$$
\|u\|_{H^{2}(\Omega)} \leq c_{m}\left\|Q_{m} u\right\|_{L_{2}(\Omega)}, \quad m=1,2 .
$$

The operators $Q_{m}, m=1,2$, are self-adjoint on $\operatorname{Dom} Q_{m} \cap H_{0}^{1}(\Omega)$,

$$
\begin{aligned}
\left(Q_{m} u, v\right)_{L_{2}(\Omega)} & =-\mu_{m}(\nabla u, \nabla v)_{L_{2}(\Omega)}-\left(\lambda_{m}+\mu_{m}\right)(\nabla \cdot u, \nabla \cdot v)_{L_{2}(\Omega)} \\
& =\left(u, Q_{m}\right)_{L_{2}(\Omega)} \quad \text { for } u, v \in \operatorname{Dom} Q_{m} \cap H_{0}^{1}(\Omega) .
\end{aligned}
$$

Moreover, the operators $-Q_{m}$ are positive on $\operatorname{Dom} Q_{m} \cap H_{0}^{1}(\Omega)$,

$$
-\left(Q_{m} u, u\right)=\mu_{m}\|\nabla u\|_{L_{2}(\Omega)}^{2}+\left(\lambda_{m}+\mu_{m}\right)\|\nabla \cdot u\|_{L_{2}(\Omega)}^{2} \geq 0 .
$$

Hence there exists the fractional power $Q_{m}^{1 / 2}$ satisfying

$$
\left(Q_{m}^{1 / 2} u, Q_{m}^{1 / 2} v\right)=\left(-Q_{m} u, v\right)_{L_{2}(\Omega)}=\left(u,-Q_{m} v\right)_{L_{2}(\Omega)}
$$

for $u, v \in \operatorname{Dom} Q_{m} \cap H_{0}^{1}(\Omega)$. 
From (1.19) and the Korn inequality

$$
d_{0}^{1 / 2}\|u\|_{H^{1}(\Omega)} \leq\|\varepsilon(u)\|_{L_{2}(\Omega)} \quad \text { for } u \in H_{0}^{1}(\Omega), d_{0}>0,
$$

it follows that

$$
\begin{aligned}
\left\|Q_{m}^{1 / 2} u\right\|_{L_{2}(\Omega)}^{2} & =\mu_{m}\|\nabla u\|_{L_{2}(\Omega)}^{2}+\left(\lambda_{m}+\mu_{m}\right)\|\nabla \cdot u\|_{L_{2}(\Omega)}^{2} \\
& =\left(A_{m} \varepsilon(u), \varepsilon(u)\right)_{L_{2}(\Omega)} \geq a_{m *}\|\varepsilon(u)\|_{L_{2}(\Omega)}^{2} \\
& \geq a_{m *} d_{0}\|u\|_{H^{1}(\Omega)}^{2} .
\end{aligned}
$$

Main Theorem. Suppose that (1.7), 1.8, $g>0$ and the assumptions of Lemma 4.1 with $A=$ const hold. Let $T \in(0, \infty), b \in L_{p, p_{0}}\left(\Omega^{T}\right)$, $g \in L_{q, q_{0}}\left(\Omega^{T}\right) \cap L_{1}\left(0, T ; L_{\infty}(\Omega)\right), u_{0} \in W_{p}^{2}(\Omega), u_{1} \in B_{p, p_{0}}^{2-2 / p_{0}}(\Omega), \theta_{0} \in$ $B_{q, q_{0}}^{2-2 / q_{0}}(\Omega), p, p_{0}, q, q_{0} \geq 4,1 / 2<\sigma \leq 1$. Assume $S \in C^{2}$. Then there exists a global solution to problem (1.1)-1.4 such that

$$
u_{, t} \in W_{p, p_{0}}^{2,1}\left(\Omega^{T}\right), \quad \theta \in W_{q, q_{0}}^{2,1}\left(\Omega^{T}\right), \quad \theta \geq \theta_{*}>0 .
$$

The paper is organized in the following way. In Section 2 we show that the property $g \geq 0$ (see [PZ1]) implies that the second law of thermodynamics holds. In Section 3 we define the spaces used, together with the corresponding imbeddings and interpolations, and we present solvability results for some parabolic initial-boundary value problems (3.1) and (3.4). Section 4 is devoted to showing a positive infimum of the temperature. In Section 5 we state some global a priori estimates. The main estimate is the Hölder continuity of temperature, which implies that $W_{q, q_{0}}^{2,1}$-theory can be applied to 1.2 . This is compatible with the results of Section 6 . Applying the method of successive approximations, in Section 6 we prove local existence of solutions to problem (1.1)-(1.4). Finally, in Section 7 we show global existence of solutions to (1.1) 1.4 by applying comparison results from Sections 5 and 6 .

2. Physical and thermodynamical background. In view of the basic thermodynamic relations, the specific internal energy $e$ and the entropy $\eta$ are related to the free energy $f$ by the formula

$$
e=f+\theta \eta, \quad \eta=-f_{, \theta} .
$$

For the free energy $f$ defined by $1.9-1.11$ this gives

$$
e=\frac{c_{v}}{\sigma+1} \theta^{\sigma+1}+\frac{1}{2} \varepsilon \cdot\left(A_{2} \varepsilon\right), \quad \eta=\frac{c_{v}}{\sigma} \theta^{\sigma}+\left(A_{2} \alpha\right) \cdot \varepsilon .
$$

As a consequence of the second law of thermodynamics expressed by the Clausius-Duhem inequality, the stress tensor $\mathbb{S}$ and the heat flux $q$ satisfy

$$
\mathbb{S}=\frac{\partial f}{\partial \varepsilon}+\theta \frac{\partial \mathcal{D}}{\partial \varepsilon_{t}}, \quad q=\frac{\partial \mathcal{D}}{\partial \nabla 1 / \theta} .
$$

and the explicit forms of $\mathbb{S}$ and $q$ are described by 1.6 and 1.15 . 
Repeating the considerations from [PZ1, Section 2] we can derive the Clausius-Duhem inequality

$$
\eta_{t}+\nabla \cdot \frac{q}{\theta} \geq \frac{g}{\theta}
$$

\section{Notation and auxiliary results}

3.1. Notation. Let $\Omega \subset \mathbb{R}^{n}, n \geq 1$, be a domain with boundary $S$. Let $\Omega^{T}=\Omega \times(0, T), S^{T}=S \times(0, T)$ with $T$ finite. By $W_{p}^{k}(\Omega), k \in \mathbb{N} \cup\{0\} \equiv \mathbb{N}_{0}$, $p \in[1, \infty)$, we denote the Sobolev space with the norm

$$
\|u\|_{W_{p}^{k}(\Omega)}=\left(\sum_{|\alpha| \leq k} \int_{\Omega}\left|D_{x}^{\alpha} u\right|^{p} d x\right)^{1 / p},
$$

where $\alpha=\left(\alpha_{1}, \ldots, \alpha_{n}\right)$ is a multi-index, $\alpha_{i} \in \mathbb{N}_{0},|\alpha|=\alpha_{1}+\cdots+\alpha_{n}$, $D_{x}^{\alpha}=\partial_{x_{1}}^{\alpha_{1}} \cdots \partial_{x_{n}}^{\alpha_{n}}$. Let $H^{k}(\Omega)=W_{2}^{k}(\Omega)$.

Next, we introduce anisotropic Lebesgue spaces $L_{p, p_{0}}\left(\Omega^{T}\right)=L_{p_{0}}(0, T$; $\left.L_{p}(\Omega)\right), p, p_{0} \in[1, \infty]$, with the norm

$$
\|u\|_{L_{p, p_{0}}\left(\Omega^{T}\right)}=\left(\int_{0}^{T}\|u(t)\|_{L_{p}(\Omega)}^{p_{0}} d t\right)^{1 / p_{0}} .
$$

Moreover, $W_{p, p_{0}}^{k, k / 2}\left(\Omega^{T}\right), k, k / 2 \in \mathbb{N}_{0}, p, p_{0} \in[1, \infty]$, is the Sobolev space with a mixed norm, which is a completion of the set of $C^{\infty}\left(\Omega^{T}\right)$-functions under the norm

$$
\|u\|_{W_{p, p_{0}}^{k, k / 2}\left(\Omega^{T}\right)}=\left(\int_{0}^{T}\left(\sum_{|\alpha|+2 a \leq k} \int_{\Omega}\left|D_{x}^{\alpha} \partial_{t}^{a} u\right|^{p} d x\right)^{p_{0} / p} d t\right)^{1 / p_{o}} .
$$

We denote by $W_{p, p_{0}}^{s, s}\left(\Omega^{T}\right), s \in \mathbb{R}_{+}, p, p_{0} \in[1, \infty]$, the Sobolev-Slobodetskiı space with the norm

$$
\begin{aligned}
\|u\|_{W_{p, p_{0}}^{s, s / 2}\left(\Omega^{T}\right)}=\sum_{|\alpha|+2 a \leq[s]}\left\|D_{x}^{\alpha} \partial_{t}^{a} u\right\|_{L_{p, p_{0}}\left(\Omega^{T}\right)} \\
+\left[\int_{0}^{T}\left(\int_{\Omega} \sum_{\Omega|\alpha|+2 a=[s]} \frac{\left|D_{x}^{\alpha} \partial_{t}^{a} u(x, t)-D_{x^{\prime}}^{\alpha} \partial_{t}^{a} u\left(x^{\prime}, t\right)\right|^{p}}{\left|x-x^{\prime}\right|^{n+p(s-[s])}} d x d x^{\prime}\right)^{p_{0} / p} d t\right]^{1 / p_{0}} \\
+\left[\int_{0}^{T} \int_{0}^{T}\left(\int_{\Omega|\alpha|+2 a=[s]} \frac{\left|D_{x}^{\alpha} \partial_{t}^{a} u(x, t)-D_{x}^{\alpha} \partial_{t^{\prime}}^{a} u\left(x, t^{\prime}\right)\right|^{p}}{\left|t-t^{\prime}\right|^{1+p(s / 2-[s / 2])}} d x\right)^{p_{0} / p} d t d t^{\prime}\right]^{1 / p_{0}},
\end{aligned}
$$

where $a \in \mathbb{N}_{0}$ and $[s]$ is the integer part of $s$.

For $s$ odd the last term in the above norm vanishes whereas for $s$ even the last two terms vanish. We also use the notation $L_{p}\left(\Omega^{T}\right)=L_{p, p}\left(\Omega^{T}\right)$, $W_{p}^{s, s / 2}\left(\Omega^{T}\right)=W_{p, p}^{s, s / 2}\left(\Omega^{T}\right)$, and so on. 
$B_{p, p_{0}}^{l}(\Omega), l \in \mathbb{R}_{+}, p, p_{0} \in[1, \infty)$, is the Besov space of functions making the following norm finite:

$$
\|u\|_{B_{p, p_{0}}^{l}(\Omega)}=\|u\|_{L_{p}(\Omega)}+\left(\sum_{i=1}^{\infty} \int_{0}^{\infty} \frac{\left\|\Delta_{i}^{m}(h, \Omega) \partial_{x_{i}}^{k} u\right\|_{L_{p}(\Omega)}^{p_{0}}}{h^{1+(l-k) p_{0}}} d h\right)^{1 / p_{0}},
$$

where $k \in \mathbb{N}_{0}, m \in \mathbb{N}, m>l-k>0, \Delta_{i}^{j}(h, \Omega) u, j \in \mathbb{N}, h \in \mathbb{R}_{+}$, is the finite difference of the order $j$ of the function $u(x)$ with respect to $x_{i}$ with

$$
\begin{aligned}
\Delta_{i}^{1}(h, \Omega) u & =\Delta_{i}(h, \Omega) u \\
& =u\left(x_{1}, \ldots, x_{i-1}, x_{i}+h, x_{i+1}, \ldots, x_{n}\right)-u\left(x_{1}, \ldots, x_{n}\right), \\
\Delta_{i}^{j}(h, \Omega) u & =\Delta_{i}(h, \Omega) \Delta_{i}^{j-1}(h, \Omega) u \text { and } \Delta_{i}^{j}(h, \Omega) u=0 \text { for } x+j h \notin \Omega .
\end{aligned}
$$

From [G] it is known that the norms of the Besov space $B_{p, p_{0}}^{l}(\Omega)$ are equivalent for different $m$ and $k$ satisfying $m>l-k>0$.

We denote by $V_{2}\left(\Omega^{T}\right)$ the space $L_{\infty}\left(0, T ; L_{2}(\Omega)\right) \cap L_{2}\left(0, T ; H^{1}(\Omega)\right)$ of functions making the following norm finite:

$$
\|u\|_{V_{2}\left(\Omega^{T}\right)}=\operatorname{ess~sup}_{t \in[0, T]}\|u(t)\|_{L_{2}(\Omega)}+\|\nabla u\|_{L_{2}\left(\Omega^{T}\right)} .
$$

Next, $V_{2}^{1,0}\left(\Omega^{T}\right)=V_{2}\left(\Omega^{T}\right) \cap C\left([0, T] ; L_{2}(\Omega)\right)$.

Let $C^{\alpha, \alpha / 2}\left(\Omega^{T}\right), \alpha \in(0,1)$, denote the anisotropic Hölder space of functions making the following norm finite:

$$
\begin{aligned}
\|u\|_{C^{\alpha, \alpha / 2}\left(\Omega^{T}\right)}= & \sup _{\Omega^{T}}|u(x, t)|+\sup _{x^{\prime}, x^{\prime \prime}, t} \frac{\left|u\left(x^{\prime}, t\right)-u\left(x^{\prime \prime}, t\right)\right|}{\left|x^{\prime}-x^{\prime \prime}\right|^{\alpha}} \\
& +\sup _{x, t^{\prime}, t^{\prime \prime}} \frac{\left|u\left(x, t^{\prime}\right)-u\left(x, t^{\prime \prime}\right)\right|}{\left|t^{\prime}-t^{\prime \prime}\right|^{\alpha / 2}} .
\end{aligned}
$$

We denote by $c$ a generic positive constant which changes its value from formula to formula and depends at most on the imbedding constants, constants of the problem, and the regularity of the boundary.

Let $\varphi=\varphi\left(\sigma_{1}, \ldots, \sigma_{k}\right), k \in \mathbb{N}$, denote a generic positive increasing function of its arguments $\sigma_{1}, \ldots, \sigma_{k}$, which may change from formula to formula.

3.2. Auxiliary results. We need the following interpolation lemma:

Lemma 3.1 (see [BIN], Ch. 4, Sect. 18]). Let $\varepsilon \in(0,1), u \in W_{p, p_{0}}^{s, s / 2}\left(\Omega^{T}\right)$, $s \in \mathbb{R}_{+}, p, p_{0} \in[1, \infty]$, and $\Omega \subset \mathbb{R}^{2}$. Let $\sigma \in \mathbb{R}_{+} \cup\{0\}$, and suppose

$$
\varkappa=\frac{2}{p}+\frac{2}{p_{0}}-\frac{2}{q}-\frac{2}{q_{0}}+|\alpha|+2 a+\sigma<s .
$$

Then $D_{x}^{\alpha} \partial_{t}^{a} u \in W_{q, q_{0}}^{\sigma, \sigma / 2}\left(\Omega^{T}\right), q \geq p, q_{0} \geq p_{0}$, and

$$
\left\|D_{x}^{\alpha} \partial_{t}^{a} u\right\|_{W_{q, q_{0}}^{\sigma, \sigma / 2}\left(\Omega^{T}\right)} \leq \varepsilon^{s-\varkappa}\|u\|_{W_{p, p_{0}}^{s, s / 2}\left(\Omega^{T}\right)}+c \varepsilon^{-\varkappa}\|u\|_{L_{p, p_{0}}\left(\Omega^{T}\right)} .
$$


As a special case of Lemma 3.1 we need

Lemma 3.2 (see [BIN, Ch. 4, Sect. 18]). Let $\varepsilon \in(0,1), u \in W_{p}^{s}(\Omega)$, $s \in \mathbb{R}_{+}, p \in[1, \infty], \Omega \subset \mathbb{R}^{2}$. Let $\sigma \in \mathbb{R}_{+} \cup\{0\}$ and suppose

$$
\varkappa=\frac{2}{p}-\frac{2}{q}+|\alpha|+\sigma<s .
$$

Then $D_{x}^{\alpha} u \in W_{q}^{\sigma}(\Omega), q \geq p$, and

$$
\left\|D_{x}^{\alpha} u\right\|_{W_{q}^{\sigma}(\Omega)} \leq \varepsilon^{s-\varkappa}\|u\|_{W_{p}^{s}(\Omega)}+c \varepsilon^{-\varkappa}\|u\|_{L_{p}(\Omega)} .
$$

We also need the following interpolation result

Lemma 3.3 (see [BIN, Ch. 3, Sect. 15]). Assume that $u \in W_{p_{2}}^{l}(\Omega) \cap$ $L_{p_{1}}(\Omega), \Omega \subset \mathbb{R}^{2}$ and

$$
\frac{2}{p}-r=(1-\theta) \frac{2}{p_{1}}+\theta\left(\frac{2}{p_{2}}-l\right) .
$$

Then

$$
\sum_{|\alpha|=r}\left\|D_{x}^{\alpha} u\right\|_{L_{p}(\Omega)} \leq c\|u\|_{W_{p_{2}}^{l}(\Omega)}^{\theta}\|u\|_{L_{p_{1}}(\Omega)}^{1-\theta} .
$$

We recall from $[\mathrm{B}]$ the trace and the inverse trace theorems for SobolevSlobodetskil spaces with mixed norm.

LEMMA 3.4.

(i) Let $u \in W_{p, p_{0}}^{s, s / 2}\left(\Omega^{T}\right), s \in \mathbb{R}_{+}, s>2 / p_{0}, p, p_{0} \in(1, \infty)$. Then $u\left(x, t_{0}\right)=\left.u(x, t)\right|_{t=t_{0}}$ for $t_{0} \in[0, T]$ belongs to $B_{p, p_{0}}^{s-2 / p_{0}}(\Omega)$ and

$$
\left\|u\left(\cdot, t_{0}\right)\right\|_{B_{p, p_{0}}^{s-2 / p_{0}(\Omega)}} \leq c\|u\|_{W_{p, p_{0}}^{s, s / 2}\left(\Omega^{T}\right)} .
$$

(ii) For a given $\tilde{u} \in B_{p, p_{0}}^{s-2 / p_{0}}(\Omega), s \in \mathbb{R}_{+}, s>2 / p_{0}, p, p_{0} \in(1, \infty)$, there exists a function $u \in W_{p, p_{0}}^{s, s / 2}\left(\Omega^{T}\right)$ such that $\left.u\right|_{t=t_{0}}=\tilde{u}$ for $t_{0} \in[0, T]$ and

$$
\|u\|_{W_{p, p_{0}}^{s, s / 2}\left(\Omega^{T}\right)} \leq c\|\tilde{u}\|_{B_{p, p_{0}}^{s-2 / p_{0}}(\Omega)} .
$$

Lemma 3.5 (see BIN, Ch. 3, Sect. 10.4 and Ch. 4, Sect. 18]). Let $\varepsilon \in(0,1), u \in W_{p, p_{0}}^{s, s / 2}\left(\Omega^{T}\right), s \in \mathbb{R}_{+}, p, p_{0} \in(1, \infty)$, and $\Omega \subset \mathbb{R}^{2}$. Let $\sigma \in \mathbb{R}_{+}$and assume

$$
\varkappa=\frac{2}{p}+\frac{2}{p_{0}}+\sigma<s .
$$

Then $u \in C^{\sigma, \sigma / 2}\left(\Omega^{t}\right)$ and

$$
\|u\|_{C^{\sigma, \sigma / 2}\left(\Omega^{t}\right)} \leq \varepsilon^{s-\varkappa}\|u\|_{W_{p, p_{0}}^{s, s / 2}\left(\Omega^{t}\right)}+c \varepsilon^{-\varkappa}\|u\|_{L_{p, p_{0}}\left(\Omega^{t}\right)} .
$$


Let us consider the problem

$$
\begin{array}{ll}
u_{t}-Q u=f & \text { in } \Omega^{T}, \\
u=0 & \text { on } S^{T}, \\
\left.u\right|_{t=0}=u_{0} & \text { in } \Omega,
\end{array}
$$

where $\Omega \subset \mathbb{R}^{2}$ and

$$
Q u=\mu \Delta u+\nu \nabla(\nabla \cdot u)
$$

with $\mu, \nu>0$. Notice that $Q$ replaces $Q_{i}$, so $\mu=\mu_{i}, \nu=\lambda_{i}+\mu_{i}, i=1,2$. Hence assumption 1.9 implies that indeed $\mu, \nu>0$.

Lemma 3.6 (parabolic system in $W_{p, p_{0}}^{2,1}\left(\Omega^{T}\right)$ [K, [PZ2, [S1, S2]).

(i) Assume that $f \in L_{p, p_{0}}\left(\Omega^{T}\right), u_{0} \in B_{p, p_{0}}^{2-2 / p_{0}}(\Omega), p, p_{0} \in(1, \infty)$ and $S \in C^{2}$. If $2-2 / p_{0}-1 / p>0$, the compatibility condition $\left.u_{0}\right|_{S}=0$ is assumed. Then there exists a unique solution to problem (3.1) such that $u \in W_{p, p_{0}}^{2,1}\left(\Omega^{T}\right)$ and

$$
\|u\|_{W_{p, p_{0}}^{2,1}\left(\Omega^{T}\right)} \leq c\left(\|f\|_{L_{p, p_{0}}\left(\Omega^{T}\right)}+\left\|u_{0}\right\|_{B_{p, p_{0}}^{2-2 / p_{0}}(\Omega)}\right)
$$

with a constant $c$ depending on $\Omega, S, p, p_{0}$.

(ii) Assume that $f=\nabla \cdot g+b, g=\left\{g_{i j}\right\}, g, b \in L_{p, p_{0}}\left(\Omega^{T}\right)$, and $u_{0} \in$ $B_{p, p_{0}}^{2-2 / p_{0}}(\Omega)$. Assume the compatibility condition

$$
\left.u_{0}\right|_{S}=0 \quad \text { if } 1-2 / p_{0}-1 / p>0 .
$$

Then there exists a unique solution to (3.1) such that $u \in W_{p, p_{0}}^{1,1 / 2}\left(\Omega^{T}\right)$ and

$$
\|u\|_{W_{p, p_{0}}^{1,1 / 2}\left(\Omega^{T}\right)} \leq c\left(\|g\|_{L_{p, p_{0}}\left(\Omega^{T}\right)}+\|b\|_{L_{p, p_{0}}\left(\Omega^{T}\right)}+\left\|u_{0}\right\|_{B_{p, p_{0}}^{2-2 / p_{0}}(\Omega)}\right)
$$

with a constant $c$ depending on $\Omega, S, p, p_{0}$.

Let us consider the problem

$$
\begin{array}{ll}
\alpha(x, t) \theta_{, t}-\Delta \theta=f & \text { in } \Omega^{T}, \\
\bar{n} \cdot \nabla \theta=0 & \text { on } S^{T}, \\
\left.\theta\right|_{t=0}=\theta_{0} & \text { in } \Omega .
\end{array}
$$

Lemma 3.7. Assume that $f \in L_{p, p_{0}}\left(\Omega^{T}\right), \theta_{0} \in B_{p, p_{0}}^{2-2 / p_{0}}(\Omega), p, p_{0} \in$ $(1, \infty)$, and $S \in C^{2}$. Assume that $\alpha \geq \alpha_{0}>0, \alpha_{0}$ is a constant, $\alpha \leq \alpha_{*}<\infty$, $\alpha_{*}$ a constant, $\alpha \in C^{\delta, \delta / 2}\left(\Omega^{T}\right), \alpha_{t} \in L_{1 / \mu, 1 / 1-\mu}\left(\Omega^{T}\right), \mu \in(0,1)$. Then there exists a solution to problem (3.4) such that $\theta \in W_{p, p_{0}}^{2,1}\left(\Omega^{T}\right)$ and

$$
\begin{aligned}
\|\theta\|_{W_{p, p_{0}}^{2,1}\left(\Omega^{T}\right)} \leq & \varphi\left(1 / \alpha_{0}, \alpha_{*},\|\alpha\|_{C^{\delta, \delta / 2}},\left\|\alpha_{, t}\right\|_{L_{1 / \mu, 1 / 1-\mu}\left(\Omega^{T}\right)}\right) \\
& \times\left(\|f\|_{L_{p, p_{0}}\left(\Omega^{T}\right)}+\left\|\theta_{0}\right\|_{B_{p, p_{0}}^{2-2 / p_{0}}(\Omega)}\right) .
\end{aligned}
$$


Proof. We prove (3.5) only, because existence follows from the regularizer technique presented in [LSU, Ch. 4 and S2]. Multiplying (3.4) 1 by $\theta$, integrating over $\Omega$ by parts and using the boundary condition $3.4 \int_{2}$ yields

$$
\frac{1}{2} \frac{d}{d t} \int_{\Omega} \alpha \theta^{2} d x+\int_{\Omega}|\nabla \theta|^{2} d x \leq\|f\|_{L_{2}}\|\theta\|_{L_{2}}+\int_{\Omega}|\alpha, t| \theta^{2} d x .
$$

By the Hölder inequality the last term on the r.h.s. of $(3.6)$ is estimated by

$$
\int_{\Omega}\left|\alpha_{, t}\right| \theta^{2} d x \leq\left\|\alpha_{, t}\right\|_{L_{\lambda_{1}}(\Omega)}\|\theta\|_{L_{2 \lambda_{2}}(\Omega)}^{2} \equiv I_{1}
$$

where $1 / \lambda_{1}+1 / \lambda_{2}=1$. Using the interpolation

$$
\|\theta\|_{L_{2 \lambda_{2}}(\Omega)} \leq c\|\nabla \theta\|_{L_{2}(\Omega)}^{\mu}\|\theta\|_{L_{2}(\Omega)}^{1-\mu}+c\|\theta\|_{L_{2}(\Omega)}
$$

with $1 / \lambda_{2}=1-\mu, \mu \in(0,1)$, we obtain, for any $\epsilon>0$,

$$
\begin{aligned}
I_{1} & \leq c\left\|\alpha_{, t}\right\|_{L_{1 / \mu}(\Omega)}\left(\|\nabla \theta\|_{L_{2}(\Omega)}^{2 \mu}\|\theta\|_{L_{2}(\Omega)}^{2(1-\mu)}+\|\theta\|_{L_{2}(\Omega)}^{2}\right) \\
& \leq \epsilon\|\nabla \theta\|_{L_{2}(\Omega)}^{2}+\varphi(1 / \epsilon)\left\|\alpha_{, t}\right\|_{L_{1 / \mu}(\Omega)}^{1 / 1-\mu}\|\theta\|_{L_{2}(\Omega)}^{2}+c\left\|\alpha_{, t}\right\|_{L_{1 / \mu}(\Omega)}\|\theta\|_{L_{2}(\Omega)}^{2} .
\end{aligned}
$$

Using (3.6) and taking $\epsilon$ sufficiently small we obtain

$$
\begin{aligned}
\frac{d}{d t} \int_{\Omega} \alpha \theta^{2} d x+\int_{\Omega}|\nabla \theta|^{2} d x \leq & \|f\|_{L_{2}(\Omega)}\|\theta\|_{L_{2}(\Omega)}+c\left\|\alpha \alpha_{, t}\right\|_{L_{1 / \mu}(\Omega)}^{1 / 1-\mu}\|\theta\|_{L_{2}(\Omega)}^{2} \\
& +c\left\|\alpha_{, t}\right\|_{L_{1 / \mu}(\Omega)}\|\theta\|_{L_{2}(\Omega)}^{2} .
\end{aligned}
$$

Since $\int_{\Omega} \theta^{2} d x \leq \frac{1}{\alpha_{0}} \int_{\Omega} \alpha \theta^{2} d x$ we derive from 3.7 the inequality

$$
\begin{aligned}
\frac{d}{d t} \int_{\Omega} \alpha \theta^{2} d x+\int_{\Omega}|\nabla \theta|^{2} d x \leq \frac{1}{\sqrt{\alpha_{0}}}\|f\|_{L_{2}(\Omega)}\left(\int_{\Omega} \alpha \theta^{2} d x\right)^{1 / 2} \\
\quad+c \frac{\|\alpha, t\|_{L_{1 / \mu}(\Omega)}^{1 / 1-\mu}}{\alpha_{0}} \int_{\Omega} \alpha \theta^{2} d x+\frac{c}{\alpha_{0}}\|\alpha, t\|_{L_{1 / \mu}(\Omega)} \int_{\Omega} \alpha \theta^{2} d x .
\end{aligned}
$$

Omitting the second term on the l.h.s. of (3.8) yields

$$
\begin{aligned}
& \frac{d}{d t}\left(\int_{\Omega} \alpha \theta^{2} d x\right)^{1 / 2} \\
& \quad \leq \frac{1}{\sqrt{\alpha_{0}}}\|f\|_{L_{2}(\Omega)}+\frac{c}{\alpha_{0}}\left(\left\|\alpha_{, t}\right\|_{L_{1 / \mu}(\Omega)}^{1 / 1-\mu}+\left\|\alpha_{, t}\right\|_{L_{1 / \mu}(\Omega)}\right)\left(\int_{\Omega} \alpha \theta^{2} d x\right)^{1 / 2} .
\end{aligned}
$$

After deriving an estimate for $\left(\int_{\Omega} \alpha \theta^{2} d x\right)^{1 / 2}$ from 3.9 , we insert it in $(3.7)$. Integrating the result with respect to time yields

$$
\begin{aligned}
\|\theta\|_{V_{2}^{0}\left(\Omega^{t}\right)} \leq & \varphi\left(1 / \alpha_{0},\left\|\alpha_{, t}\right\|_{L_{1 / \mu, 1 / 1-\mu}\left(\Omega^{t}\right)},\|f\|_{L_{2}\left(\Omega^{t}\right)},\left\|\theta_{0}\right\|_{L_{2}(\Omega)}\right) . \\
& \times\left[\|f\|_{L_{2}\left(\Omega^{t}\right)}+\left\|\theta_{0}\right\|_{L_{2}(\Omega)}\right], \quad t \leq T .
\end{aligned}
$$


Using a partition of unity $\zeta^{(k)}(x, t)$ such that $\bigcup_{k} \operatorname{supp} \zeta^{(k)}(x, t)=\Omega \times(0, T)$ and introducing the notation $u^{(k)}=u \zeta^{(k)}$ we obtain from 3.4 the following problem, localized to $\operatorname{supp} \zeta^{(k)}$ :

$$
\begin{aligned}
& \alpha\left(\xi^{(k)}, t^{(k)}\right) \theta_{, t}^{(k)}-\Delta \theta^{(k)}=\left[\alpha\left(\xi^{(k)}, t^{(k)}\right)-\alpha(x, t)\right] \theta_{, t}^{(k)} \\
& +f^{(k)}+\alpha \zeta_{, t}^{(k)} \theta-2 \nabla \theta \nabla \zeta^{(k)}-\theta \Delta \zeta^{(k)}, \\
& \bar{n} \cdot \nabla \theta^{(k)}=\bar{n} \cdot \nabla \zeta^{(k)} \theta, \\
& \left.\theta^{(k)}\right|_{t=0}=\left.\theta_{0} \zeta^{(k)}\right|_{t=0},
\end{aligned}
$$

where $\left(\xi^{(k)}, t^{(k)}\right)$ is a "middle" point of $\operatorname{supp} \zeta^{(k)}$. In view of [K, S1] and 3.10] we have

$$
\begin{aligned}
\left\|\theta^{(k)}\right\|_{W_{p, p_{0}}^{2,1}\left(\Omega^{T}\right)} \leq & \varphi\left(1 / \alpha_{0}, \alpha^{*},\|\alpha\|_{C^{\delta, \delta / 2}},\left\|\alpha_{, t}\right\|_{L_{1 / \mu, 1 / 1-\mu}\left(\Omega^{t}\right)}\right) \\
& \times\left[\left\|f^{(k)}\right\|_{L_{p, p_{0}}\left(\Omega^{T}\right)}+\left\|\left.\theta^{(k)}\right|_{t=0}\right\|_{B_{p, p_{0}}^{2-2 / p_{0}}(\Omega)}\right]
\end{aligned}
$$

Summing up over all subdomains $\operatorname{supp} \zeta^{(k)}(x, t)$ we obtain 3.5 .

4. Lower bound for temperature. The existence of the lower positive bound on the temperature is important in getting an a priori global estimate in this paper. We follow [PZ1, proof of Lemma 4.1], but the argument is different.

Lemma 4.1. Assume that $(1.2)$ holds, $g, \theta_{0}>0$ and $A=A(\varepsilon)$. Let

$$
\sigma=1 \quad \text { and } \quad a_{1}(t)=\frac{1}{2 a_{1 *}} \sup _{\Omega}|A(\varepsilon)|^{2},
$$

where $a_{1 *}$ is introduced in $(1.19)$. Then for sufficiently regular solutions to problem 1.1 -1.4 we have

$$
\theta(t) \geq \theta_{0} \exp \left(-\int_{0}^{t} \frac{a_{1}\left(t^{\prime}\right)}{c_{v}} d t^{\prime}\right) \equiv \theta_{*} .
$$

Let now

$$
\sigma<1 \quad \text { and } \quad a_{2}(t)=\frac{1}{2 a_{1 *}} \lim _{\varrho \rightarrow \infty}\|A\|_{\frac{L^{((\varrho-(\sigma+1))}}{2-(\sigma+1)}}^{2}(\Omega) .
$$

Then for a sufficiently regular solution to (1.1) 1.4 we have

$$
\theta(t) \geq\left[\frac{1}{\int_{0}^{t} \frac{a_{2}\left(t^{\prime}\right)}{c_{v}} d t^{\prime}+\left(\frac{1}{\theta_{0}}\right)^{1-\sigma}}\right]^{1 /(1-\sigma)} \equiv \theta_{*} .
$$


Proof. Multiplying 1.2 by $-\theta^{-\varrho}$ and integrating over $\Omega$ yields

$$
\begin{aligned}
-c_{v} \int_{\Omega} \theta^{\sigma-\varrho} \theta_{, t} d x+\varkappa \int_{\Omega} \theta^{-\varrho} \Delta \theta d x+\int_{\Omega}\left(A_{1} \varepsilon_{,}\right) \cdot \varepsilon_{, t} \theta^{-\varrho} d x \\
\quad+\int_{\Omega} g \theta^{-\varrho} d x+\int_{\Omega} A(\varepsilon) \cdot \varepsilon_{, t} \theta^{1-\varrho} d x=0 .
\end{aligned}
$$

Now we examine the terms in (4.4) one by one. The first term is equal to

$$
\frac{c_{v}}{\varrho-(\sigma+1)} \frac{d}{d t} \int_{\Omega} \frac{d x}{\theta^{\varrho-(\sigma+1)}}
$$

The second term equals

$$
\frac{4 \varkappa \varrho}{(\varrho-1)^{2}} \int_{\Omega}\left|\nabla \frac{1}{\theta^{(\varrho-1) / 2}}\right|^{2} d x .
$$

In view of 1.19 the third term is bounded from below by

$$
a_{1 *} \int_{\Omega} \frac{|\varepsilon, t|^{2}}{\theta^{\varrho}} d x
$$

The fourth term is positive because $g>0$. From the Cauchy inequality the last term in 4.4 is bounded by

$$
\frac{a_{1 *}}{2} \int_{\Omega} \frac{\left|\varepsilon_{, t}\right|^{2}}{\theta^{\varrho}} d x+\frac{1}{2 a_{1 *}} \int_{\Omega}|A(\varepsilon)|^{2} \theta^{2-\varrho} d x .
$$

In view of the above considerations, (4.4) takes the form

$$
\begin{aligned}
\frac{c_{v}}{\varrho-(\sigma+1)} & \frac{d}{d t} \int_{\Omega} \frac{1}{\theta^{\varrho-(\sigma+1)}} d x+\frac{4 \varkappa \varrho}{(\varrho-1)^{2}} \int_{\Omega}\left|\nabla \frac{1}{\theta^{(\varrho-1) / 2}}\right|^{2} d x \\
& +\frac{a_{1 *}}{2} \int_{\Omega} \frac{\left|\varepsilon_{, t}\right|^{2}}{\theta^{\varrho}} d x+\int_{\Omega} \frac{g}{\theta^{\varrho}} d x \leq \frac{1}{2 a_{1 *}} \int_{\Omega}|A(\varepsilon)|^{2} \theta^{2-\varrho} d x,
\end{aligned}
$$

where $\varrho$ is assumed to be large. Set

$$
X=\left(\int_{\Omega} \frac{1}{\theta^{\varrho-(\sigma+1)}} d x\right)^{\frac{1}{\varrho-(\sigma+1)}} .
$$

Using (4.6) and assuming that $a_{1}(t)=\frac{1}{2 a_{1 *}} \sup _{\Omega}|A(\varepsilon)|^{2}$ we obtain

$$
\frac{c_{v}}{\varrho-(\sigma+1)} \frac{d}{d t} X^{\varrho-(\sigma+1)} \leq a_{1}(t) \int_{\Omega} \frac{1}{\theta^{\varrho-2}} d x .
$$

Setting $\sigma=1$ we obtain from 4.7 the inequality

$$
c_{v} \frac{d}{d t} X \leq a_{1}(t) X, \quad \text { so } \quad X(t) \leq X(0) \exp \int_{0}^{t} \frac{a_{1}\left(t^{\prime}\right)}{c_{v}} d t .
$$


Hence letting $\varrho \rightarrow \infty$ gives

$$
\theta(t) \geq \theta(0) \exp \left[-\int_{0}^{t} \frac{a_{1}\left(t^{\prime}\right)}{c_{v}} d t^{\prime}\right],
$$

which yields 4.2 ).

Let us now consider the case $\sigma<1$. Then 4.5 takes the form

$$
\begin{aligned}
& \frac{c_{v}}{\varrho-(\sigma+1)} \frac{d}{d t} X^{\varrho-(\sigma+1)} \\
& \leq \frac{1}{2 a_{1 *}}\|A\|_{L_{\frac{2(\varrho-(\sigma+1))}{2-(\sigma+1)}}^{2}}\left(\int_{\Omega}\left|\frac{1}{\theta}\right|^{\varrho-(\sigma+1)} d x\right)^{\frac{\varrho-2}{\varrho-(\sigma+1)}} \equiv a_{2}(t, \varrho) X^{\varrho-2} .
\end{aligned}
$$

Hence

$$
\frac{d}{d t} X \leq \frac{a_{2}(t, \varrho)}{c_{v}} X^{\sigma} \text { and } \frac{1}{1-\sigma} \frac{d}{d t} X^{1-\sigma} \leq \frac{a_{2}(t, \varrho)}{c_{v}}
$$

so

$$
X^{1-\sigma}(t) \leq \int_{0}^{t} \frac{a_{2}\left(t^{\prime}, \varrho\right)}{c_{v}} d t^{\prime}+X^{1-\sigma}(0) .
$$

Letting $\varrho \rightarrow \infty$ we get

$$
\theta(t) \geq\left[\frac{1}{\int_{0}^{t}\left(a_{2} / c_{v}\right) d t^{\prime}+\left(1 / \theta_{0}\right)^{1-\sigma}}\right]^{1 /(1-\sigma)} .
$$

This implies 4.3 and concludes the proof.

5. A priori estimates. In this section we give estimates under the assumption that there exists a sufficiently regular local solution to problem (1.1)-(1.4). The proofs of these estimates are given in [GZ4]. Moreover, Lemma 4.1 implies existence of a constant $\theta_{*}>0$ such that

$$
\theta(x, t) \geq \theta_{*}, \quad t \leq T,
$$

where $T$ is the time of local existence.

Lemma 5.1. Assume that $u_{1} \in L_{2}(\Omega), u_{0} \in H^{1}(\Omega), \theta_{0} \in L_{\sigma+1}(\Omega)$, $b \in L_{2}\left(\Omega^{t}\right), 0<g \in L_{1}\left(\Omega^{t}\right), t \leq T$. Then solutions to problem $1.1-1.4$ satisfy

$$
\begin{aligned}
& \left\|u_{, t}(t)\right\|_{L_{2}(\Omega)}^{2}+\|u(t)\|_{H^{1}(\Omega)}^{2}+\|\theta(t)\|_{L_{\sigma+1}(\Omega)}^{\sigma+1} \\
\leq & c(t)\left(\|b\|_{L_{2}\left(\Omega^{t}\right)}^{2}+\|g\|_{L_{1}\left(\Omega^{t}\right)}^{2}+\left\|u_{1}\right\|_{L_{2}(\Omega)}^{2}+\left\|u_{0}\right\|_{H^{1}(\Omega)}^{2}+\left\|\theta_{0}\right\|_{L_{\sigma+1}(\Omega)}^{\sigma+1}\right) \\
\equiv & c_{1}(t), \quad t \leq T,
\end{aligned}
$$

where $c(t)$ is an increasing function. 
Lemma 5.2. Assume that $u_{1} \in H^{1}(\Omega), b \in L_{2,2}\left(\Omega^{t}\right), \theta_{0} \in L_{\sigma+2}(\Omega)$, $g \in L_{1}\left(0, t ; L_{(\sigma+1) / \sigma}(\Omega)\right), u_{0} \in W_{2}^{2}(\Omega), \sigma>1 / 2$. Then

$$
\int_{\Omega} \theta^{\sigma+2} d x+\|\nabla \theta\|_{L_{2}\left(\Omega^{t}\right)}^{2} \leq c\left(t, c_{2}\right),
$$

where

$$
\begin{aligned}
c_{2}= & \left\|u_{1}\right\|_{L_{2}(\Omega)}+\|b\|_{L_{2}\left(\Omega^{t}\right)}+\left\|\theta_{0}\right\|_{L_{\sigma+2}(\Omega)} \\
& +\|g\|_{L_{1}\left(0, t ; L_{(\sigma+1) / \sigma}(\Omega)\right)}+\left\|u_{0}\right\|_{W_{2}^{2}(\Omega)} .
\end{aligned}
$$

We recall important inequalities necessary to derive a global a priori estimate.

Applying Lemma 3.6(ii) to problem (1.1) (1.4) yields

$$
\begin{aligned}
& \quad\left\|\varepsilon_{, t}\right\|_{L_{p, r}\left(\Omega^{t}\right)} \leq c\left\|u_{, t}\right\|_{W_{p, r}^{1,1 / 2}\left(\Omega^{t}\right)} \\
& \leq c\left[\|\varepsilon\|_{L_{p, r}\left(\Omega^{t}\right)}+\|\theta\|_{L_{p, r}\left(\Omega^{t}\right)}+\|b\|_{L_{p, r}\left(\Omega^{t}\right)}+\left\|u_{1}\right\|_{B_{p, r}^{1-2 / r}(\Omega)}+\left\|u_{0}\right\|_{W_{p}^{1}(\Omega)}\right] .
\end{aligned}
$$

Hence, the Gronwall lemma implies

$$
\left\|\varepsilon_{, t}\right\|_{L_{p, r}\left(\Omega^{t}\right)} \leq c(t)\left[\|\theta\|_{L_{p, r}\left(\Omega^{t}\right)}+\|b\|_{L_{p, r}\left(\Omega^{t}\right)}+\left\|u_{1}\right\|_{B_{p, r}^{1-2 / r}(\Omega)}+\left\|u_{0}\right\|_{W_{p}^{1}(\Omega)}\right] .
$$

Moreover, applying Lemma 3.6(i) to (1.1), 1.3 $\left.)_{1}, 1.4\right)_{1,2}$ and the Gronwall inequality we obtain

$$
\begin{aligned}
& \left\|\varepsilon_{, t}\right\|_{W_{p, r}^{1,1 / 2}\left(\Omega^{t}\right)} \\
& \quad \leq c(t)\left[\|\nabla \theta\|_{L_{p, r}\left(\Omega^{t}\right)}+\|b\|_{L_{p, r}\left(\Omega^{t}\right)}+\left\|u_{1}\right\|_{B_{p, r}^{2-2 / r}(\Omega)}+\left\|u_{0}\right\|_{W_{p}^{2}(\Omega)}\right] .
\end{aligned}
$$

From Lemma 5.2 we have

$$
\begin{aligned}
& \theta \in L_{\infty}\left(0, t ; L_{\sigma+2}(\Omega)\right), \\
& \theta \in L_{2}\left(0, t ; L_{q}(\Omega)\right), \quad q \in(1, \infty) .
\end{aligned}
$$

In view of (5.7) inequality (5.4) implies

$$
\begin{aligned}
\left\|\varepsilon_{, t}\right\|_{L_{\sigma+2, r}\left(\Omega^{t}\right)} & \leq c\left[c\left(c_{2}\right)+\|b\|_{L_{\sigma+2, r}\left(\Omega^{t}\right)}+\left\|u_{1}\right\|_{B_{\sigma+2, r}^{1-1 / r}(\Omega)}+\left\|u_{0}\right\|_{W_{\sigma+2}^{1}(\Omega)}\right] \\
& \equiv c_{3}, \quad r \in(1, \infty) .
\end{aligned}
$$

LemmA 5.3. Assume that $b \in L_{2,2}\left(\Omega^{t}\right)$ and $u_{1} \in B_{2,2}^{1 / 2}(\Omega)$. Then (5.10) $\left\|\varepsilon_{, t}\right\|_{L_{p_{1}, r_{1}}\left(\Omega^{t}\right)} \leq c\left(1+\|b\|_{L_{2,2}\left(\Omega^{t}\right)}+\left\|u_{1}\right\|_{B_{2,2}^{1}(\Omega)}+\left\|u_{0}\right\|_{H^{2}(\Omega)}\right) \equiv c_{5}$, where $r_{1}, p_{1}$ are such that

$$
1 \leq 2\left(\frac{1}{p_{1}}+\frac{1}{r_{1}}\right) .
$$


From (5.3) and (5.6) we have

$$
\left\|\varepsilon_{, t}\right\|_{W_{2,2}^{1,1 / 2}\left(\Omega^{t}\right)} \leq c\left(1+\|b\|_{L_{2,2}\left(\Omega^{t}\right)}+\left\|u_{1}\right\|_{B_{2,2}^{1}(\Omega)}+\left\|u_{0}\right\|_{H^{2}(\Omega)}\right) .
$$

Setting $r_{1}=2$ we obtain from 5.12 the estimate

$$
\left\|\varepsilon_{, t}\right\|_{L_{p, 2}\left(\Omega^{t}\right)} \leq c_{3}
$$

where $p$ is an arbitrary finite number.

Lemma 5.4. Assume that $\theta_{0} \in L_{s+\sigma+1}(\Omega), g \in L_{1}\left(0, t ; L_{\frac{s+\sigma+1}{\sigma+1}}(\Omega)\right)$, and $s \in(1, \infty)$. Then

$$
\begin{aligned}
\|\theta\|_{L_{s+\sigma+1}(\Omega)} \leq & c\left(c_{2}, c_{3}\right) \\
& +c\|g\|_{L_{1}\left(0, t ; L_{\frac{s+\sigma+1}{\sigma+1}}(\Omega)\right)}+c\left\|\theta_{0}\right\|_{L_{s+\sigma+1}(\Omega)} \equiv c_{4} .
\end{aligned}
$$

Lemma 5.5. Assume that $b \in L_{4}\left(\Omega^{t}\right), u_{1} \in B_{4,4}^{3 / 2}(\Omega), u_{0} \in W_{4}^{2}(\Omega)$, $g \in L_{2}\left(0, t ; L_{4}(\Omega)\right), \theta_{0} \in L_{4}(\Omega), \theta \geq \theta_{*}>0$. Then

$$
\left\|\varepsilon_{, t}\right\|_{L_{2}\left(0, t ; L_{\infty}(\Omega)\right)} \leq c_{5}
$$

where $c_{5}$ depends on all norms from the assumption.

Lemma 5.6. Assume that $g \in L_{1}\left(0, T ; L_{\infty}(\Omega)\right), \varepsilon_{, t} \in L_{2}\left(0, T ; L_{\infty}(\Omega)\right)$, $\theta_{0} \in L_{\infty}(\Omega), \theta \geq \theta_{*}>0$. Then

(5.16) $\|\theta\|_{L_{\infty}\left(\Omega^{t}\right)} \leq c\left(1 / \theta_{*}\right)|\Omega|\left[c_{5}+c_{5}^{2}+\|g\|_{L_{1}\left(0, t ; L_{\infty}(\Omega)\right)}+\left\|\theta_{0}\right\|_{L_{\infty}(\Omega)}\right] \equiv c_{6}$.

To prove the Hölder continuity of the temperature we follow the method of [LSU, Ch. 2, Sect. 7]. For this purpose we recall the space $\mathcal{B}_{2}\left(\Omega^{T}, M, \gamma, r, \delta, \varkappa\right)$, $\Omega^{T}=\Omega \times(0, T), \Omega \subset \mathbb{R}^{n}$ and $M, \gamma, r, \delta, \varkappa$ are positive constants.

Definition 5.7. We say that $u \in \mathcal{B}_{2}\left(\Omega^{T}, M, \gamma, r, \delta, \varkappa\right)$ if

(1) $u \in V_{2}^{1,0}\left(\Omega^{T}\right)$,

(2) $\operatorname{ess} \sup _{\Omega^{T}}|u| \leq M$

(3) the function $w(x, t)=\mp u(x, t)$ satisfies the inequalities

$$
\begin{aligned}
\max _{t_{0} \leq t \leq t_{0}+\tau} \|(\omega-k)_{+} & \left.\|_{L_{2}\left(B_{\varrho}-\sigma_{1} \varrho\right.}^{2}\left(x_{0}\right)\right) \\
+ & \leq\left\|(\omega-k)_{+}\left(\cdot, t_{0}\right)\right\|_{L_{2}\left(B_{\varrho}\left(x_{0}\right)\right)}^{2} \\
& +\gamma\left[\left(\sigma_{1} \varrho\right)^{-2}\left\|(\omega-k)_{+}\right\|_{L_{2}(Q(\varrho, \tau))}^{2}+\mu^{\frac{2}{r}(1+\varkappa)}(k, \varrho, \tau)\right]
\end{aligned}
$$

and

$$
\begin{aligned}
& \left\|(\omega-k)_{+}\right\|_{V_{0}\left(Q\left(\varrho-\sigma_{1} \varrho, \tau-\sigma_{2} \tau\right)\right)}^{2} \\
& \quad \leq \gamma\left[\left(\left(\sigma_{1} \varrho\right)^{-2}+\left(\sigma_{2} \tau\right)^{-1}\right)\left\|(\omega-k)_{+}\right\|_{L_{2}(Q(\varrho, \tau))}^{2}+\mu^{\frac{2}{r}(1+\varkappa)}(k, \varrho, \tau)\right] .
\end{aligned}
$$

Here the following notation is used:

$$
\begin{aligned}
& (\omega-k)_{+}=\max \{\omega-k, 0\}, \quad k>0, \\
& B_{\varrho}\left(x_{0}\right)=\left\{x \in \Omega:\left|x-x_{0}\right|<\varrho\right\}, \\
& Q(\varrho, \tau)=B_{\varrho}\left(x_{0}\right) \times\left(t_{0}, t_{0}+\tau\right),
\end{aligned}
$$


and $\varrho, \tau$ are arbitrary positive numbers, $\sigma_{1}, \sigma_{2} \in(0,1)$ and $k$ is a positive number such that

$$
\underset{Q(\varrho, \tau)}{\operatorname{ess} \sup } \omega(x, t)-k<\delta .
$$

Moreover, $V_{2}^{1,0}\left(\Omega^{T}\right)$ is defined in Section 3 .

$$
\mu(k, \varrho, \tau)=\int_{t_{0}}^{t_{0}+\tau} \operatorname{meas}^{r / q} A_{k, \varrho}(t) d t,
$$

where $A_{k, \varrho}(t)=\left\{x \in B_{\varrho}\left(x_{0}\right): \omega(x, t)>k\right\}$, and the positive numbers $q, r$ are linked by the relation $1 / r+n /(2 q)=n / 4$.

Lemma 5.8. Assume $0<\theta_{*} \leq \theta$, where $\theta_{*}$ is defined by (4.1) and (4.2), respectively. Let $M \equiv\|\theta\|_{L_{\infty}\left(\Omega^{T}\right)} \leq c_{6}($ see $(5.16))$. Let $\sup _{\Omega} \theta_{0}(x)<k$ and $M-k<\delta$ with some $\delta>0$. Let $\varepsilon_{t} \in L_{2 \lambda}\left(\Omega^{T}\right), g \in L_{\lambda}\left(\Omega^{T}\right), \lambda=\frac{1}{1-\frac{2}{r}(1+\varkappa)}$, $1 / r+1 / q=1 / 2, \varkappa>0$. Then

$$
\theta \in \mathcal{B}_{2}\left(\Omega^{T}, M, \gamma, r, \delta, \varkappa\right) .
$$

Remark 5.9. By the imbedding (see [LSU, Ch. 2 Theorem 7.1])

$$
\mathcal{B}_{2}\left(\Omega^{T}, M, \gamma, r, \delta, k\right) \subset C^{\alpha, \alpha / 2}\left(\Omega^{T}\right), \quad \alpha \in(0,1),
$$

it follows from (5.17) that

$$
\theta \in C^{\alpha, \alpha / 2}\left(\Omega^{T}\right),
$$

where $\alpha$ depends on $M, \gamma, r, \delta, \varkappa$.

REMARK 5.10. In view of (5.14) and (5.5) we have $\theta, \varepsilon_{t} \in L_{p, r}\left(\Omega^{T}\right)$, $p, r \in(1, \infty)$. Using this and the Hölder continuity of $\theta$ we get for solutions to problem $1.2,(1.4)_{3},(1.3)_{2}$ the estimate

$$
\|\theta\|_{W_{q, q_{0}}^{2,1}\left(\Omega^{T}\right)} \leq \varphi\left(c_{4}, c_{6}, c_{0}\right),
$$

where $q, q_{0} \in(1, \infty)$. Hence

$$
\nabla \theta \in L_{r, r_{0}}\left(\Omega^{T}\right),
$$

where

$$
\frac{2}{q}+\frac{2}{q_{0}}-\frac{2}{r}-\frac{2}{r_{0}} \leq 1,
$$

For $q=q_{0}$ and $r=r_{0}$ condition (5.21) implies

$$
\frac{4}{q}-\frac{4}{r} \leq 1 \text {. }
$$

Since $q$ can be an arbitrary number from $(1, \infty)$, the same can be said about $r$. 
Similarly, for solutions to problem (1.1), 1.3$)_{1},(1.4)_{1,2}$ we have

$$
\|u\|_{W_{p, p_{0}}^{2,1}\left(\Omega^{T}\right)} \leq \varphi\left(c_{0}, c_{8}\right)
$$

where $p, p_{0} \in(1, \infty)$.

We are not interested in increasing the regularity of solutions to problem (1.1)-(1.6) as much as possible. We just need enough regularity that the existence of local solutions and that the local solution may be extended in time to get global existence.

6. Local existence. To prove local existence of solutions to problem (1.1) - 1.4 we use the following successive approximations:

$$
\begin{aligned}
& u_{(n+1), t t}-\nabla \cdot\left(A_{1} \varepsilon\left(u_{(n+1), t}\right)\right)=\nabla \cdot\left[A_{2} \varepsilon\left(u_{(n)}\right)+A \theta_{(n)}\right]+b \quad \text { in } \Omega^{T}, \\
& c_{v} \theta_{(n)}^{\sigma} \theta_{(n+1), t}-\varkappa \Delta \theta_{(n+1)} \\
& \quad=\theta_{(n)} A \varepsilon\left(u_{(n), t}\right)+\left(A_{1} \cdot \varepsilon\left(u_{(n), t}\right) \cdot \varepsilon\left(u_{(n), t}\right)+g \quad \text { in } \Omega^{T},\right. \\
& u_{(n+1)}=0, \quad \bar{n} \cdot \nabla \theta_{(n+1)}=0 \quad \text { on } S^{T}, \\
& \left.u_{(n+1)}\right|_{t=0}=u_{0},\left.\quad u_{(n+1), t}\right|_{t=0}=u_{1},\left.\quad \theta_{(n+1)}\right|_{t=0}=\theta_{0} \quad \text { in } \Omega,
\end{aligned}
$$

where $u_{(n)}, \theta_{(n)}$ are treated as given.

Moreover, the zero approximations $\left(u_{(0)}, \theta_{(0)}\right)$ are constructed by an extension of the initial data in such a way that

$$
\begin{aligned}
& \left.u_{(0)}\right|_{t=0}=u_{0},\left.\quad u_{(0), t}\right|_{t=0}=u_{1},\left.\quad \theta_{(0)}\right|_{t=0}=\theta_{0} \quad \text { in } \Omega, \\
& u_{(0)}=0, \quad \bar{n} \cdot \nabla \theta_{(0)}=0 \quad \text { on } S^{T} .
\end{aligned}
$$

First we show uniform boundedness of the sequence $\left\{u_{(n)}, \theta_{(n)}\right\}$.

Lemma 6.1. Suppose that $X_{0}(t)=\left\|u_{(0), t^{\prime}}\right\|_{W_{p, p_{0}}^{2,1}\left(\Omega^{t}\right)}+\left\|\theta_{(0)}\right\|_{W_{q, q_{0}}^{2,1}\left(\Omega^{t}\right)}<\infty$, where $u_{(0)}, \theta_{(0)}$ are introduced by (6.5). Suppose that $D(t)=\left\|u_{0}\right\|_{W_{p}^{2}(\Omega)}+$ $\left\|u_{1}\right\|_{B_{p, p_{0}}^{2-2 / p_{0}}(\Omega)}+\left\|\theta_{0}\right\|_{B_{q, q_{0}}^{2-2 / q_{0}}(\Omega)}+\|b\|_{L_{p, p_{0}}\left(\Omega^{t}\right)}+\|g\|_{L_{q, q_{0}}\left(\Omega^{t}\right)}<\infty$. Let $1 / p+$ $1 / p_{0}<1,1 / q+1 / q_{0}<1, q_{0}>2$. Assume that there exists a constant $\bar{A}$ and time $t$ such that $X_{0}(t) \leq \bar{A}, \varphi_{1}\left(t^{\alpha} \bar{A}, D(t)\right) \leq \bar{A}, \alpha>0$ and $\varphi_{1}$ is introduced in 6.34, and $c t^{\sigma / 2} A \leq \theta_{*}, \sigma>0, \theta_{*}=\min _{\Omega} \theta_{0}$. Then

(6.7) $\quad X_{n}(t)=\left\|u_{(n), t^{\prime}}\right\|_{W_{p}^{2, p_{0}}\left(\Omega^{t}\right)}+\left\|\theta_{(n)}\right\|_{W_{q, q_{0}}^{2,1}\left(\Omega^{t}\right)} \leq \bar{A} \quad$ for any $n \in \mathbb{N}$.

Proof. Applying Lemma 3.6 to problem 6.1), 6.3 1 , 6.4 1,2 yields

$$
\begin{aligned}
\left\|u_{(n+1), t^{\prime}}\right\|_{W_{p, p_{0}}^{2,1}\left(\Omega^{t}\right)} \leq c\left[\| \nabla^{2} u_{(n)}\right. & \left\|_{L_{p, p_{0}}\left(\Omega^{t}\right)}+\right\| \nabla \theta_{(n)} \|_{L_{p, p_{0}}\left(\Omega^{t}\right)} \\
& \left.+\|b\|_{L_{p, p_{0}}\left(\Omega^{t}\right)}+\left\|u_{1}\right\|_{B_{p, p_{0}}^{2-2 / p_{0}}(\Omega)}\right],
\end{aligned}
$$

where $c$ does not depend on $t$. With the use of the formula

$$
u_{(n)}(x, t)=\int_{0}^{t} u_{(n), t^{\prime}}\left(x, t^{\prime}\right) d t^{\prime}+u_{0}(x)
$$


the first term on the r.h.s. of 6.8 is estimated by

$$
\begin{aligned}
\left\|\nabla^{2} u_{(n)}\right\|_{L_{p, p_{0}}\left(\Omega^{t}\right)} & \leq\left\|\int_{0}^{t} \nabla^{2} u_{(n), t^{\prime}}\left(x, t^{\prime}\right) d t^{\prime}\right\|_{L_{p, p_{0}}\left(\Omega^{t}\right)}+t^{1 / p_{0}}\left\|\nabla^{2} u_{0}\right\|_{L_{p}(\Omega)} \\
& \leq t\left\|\nabla^{2} u_{(n), t^{\prime}}\right\|_{L_{p, p_{0}}\left(\Omega^{t}\right)}+t^{1 / p_{0}}\left\|\nabla^{2} u_{0}\right\|_{L_{p}(\Omega)} .
\end{aligned}
$$

Employing 6.9 in 6.8 we get

$$
\begin{aligned}
& \left\|u_{(n+1), t}\right\|_{W_{p, p_{0}}^{2,1}\left(\Omega^{t}\right)} \leq c\left\|\nabla \theta_{(n)}\right\|_{L_{p, p_{0}}\left(\Omega^{t}\right)}+t\left\|\nabla^{2} u_{(n), t^{\prime}}\right\|_{L_{p, p_{0}}\left(\Omega^{t}\right)} \\
& +c\left(t^{1 / p_{0}}\left\|\nabla^{2} u_{0}\right\|_{L_{p}(\Omega)}+\|b\|_{L_{p, p_{0}}\left(\Omega^{t}\right)}+\left\|u_{1}\right\|_{B_{p, p_{0}}^{2-2 / p_{0}}(\Omega)}\right) .
\end{aligned}
$$

Next we examine problem $6.2,(6.3)_{2},(6.4)_{3}$. By Lemma 3.7 there exists a solution to this problem and the solution satisfies

$$
\begin{aligned}
& \left\|\theta_{(n+1)}\right\|_{W_{q, q_{0}}^{2,1}\left(\Omega^{t}\right)} \\
\leq & \varphi\left(\sup _{\Omega^{t}} \frac{1}{\theta_{(n)}} \sup _{\Omega^{t}} \theta_{(n)},\left\|\theta_{(n)}\right\|_{C^{\sigma, \sigma / 2}\left(\Omega^{t}\right)},\left\|\theta_{(n), t^{\prime}}\right\|_{L_{2}\left(\Omega^{t}\right)}\right) \\
\times & {\left[\left\|\theta_{(n)} \varepsilon\left(u_{(n), t^{\prime}}\right)\right\|_{L_{q, q_{0}}\left(\Omega^{t}\right)}+\left\|\left(A_{1} \varepsilon\left(u_{(n), t^{\prime}}\right)\right) \cdot \varepsilon\left(u_{(n), t^{\prime}}\right)\right\|_{L_{q, q_{0}}\left(\Omega^{t}\right)}\right.} \\
& \left.+\|g\|_{L_{q, q_{0}}\left(\Omega^{t}\right)}+\left\|\theta_{0}\right\|_{B_{q, q_{0}}^{2-2 / q_{0}}(\Omega)}\right] .
\end{aligned}
$$

First we estimate the arguments of $\varphi$. Using Lemma 3.1 we have

$$
\begin{aligned}
\left\|\theta_{(n)}\right\|_{L_{\infty}\left(\Omega^{t}\right)} & \leq \delta\left\|\theta_{(n)}\right\|_{W_{q, q_{0}}^{2,1}\left(\Omega^{t}\right)}+\varphi(1 / \delta)\left\|\theta_{(n)}\right\|_{L_{q, q_{0}}\left(\Omega^{t}\right)} \\
& \leq c_{3}\left(t^{\alpha}\left\|\theta_{(n)}\right\|_{W_{q, q_{0}}^{2,1}\left(\Omega^{t}\right)}+\left\|\theta_{0}\right\|_{L_{q}(\Omega)}\right)
\end{aligned}
$$

under the restriction

$$
\frac{1}{q}+\frac{1}{q_{0}}<1
$$

In view of Lemma 3.5 we have

$$
\begin{aligned}
\left\|\theta_{(n)}\right\|_{C^{\sigma, \sigma / 2}\left(\Omega^{t}\right)} & \leq \delta\left\|\theta_{(n)}\right\|_{W_{q, q_{0}}^{2,1}\left(\Omega^{t}\right)}+\varphi(1 / \delta)\left\|\theta_{(n)}\right\|_{L_{q, q_{0}}\left(\Omega^{t}\right)} \\
& \leq c_{4}\left(t^{\alpha}\left\|\theta_{(n)}\right\|_{W_{q, q_{0}}^{2,1}\left(\Omega^{t}\right)}+\left\|\theta_{0}\right\|_{L_{q}(\Omega)}\right),
\end{aligned}
$$

for some $\alpha>0$, under the condition

$$
\frac{2}{q}+\frac{2}{q_{0}}+\sigma<2
$$

Assuming that $q_{0}>2$ we get

$$
\left\|\theta_{(n), t^{\prime}}\right\|_{L_{2}\left(\Omega^{t}\right)} \leq t^{1 / 2-1 / q_{0}}\left\|\theta_{(n), t^{\prime}}\right\|_{L_{q, q_{0}}\left(\Omega^{t}\right)} .
$$

To estimate $\theta_{(n)}$ from below we consider

$$
\theta_{(n)}=\theta_{(n)}-\theta_{0}+\theta_{0} \geq \theta_{*}-\left|\theta_{(n)}-\theta_{0}\right| \geq \theta_{*}-\sup _{x \in \Omega}\left\|\theta_{(n)}-\theta_{0}\right\|_{C^{\sigma / 2}(0, t)} t^{\sigma / 2} \equiv I_{1},
$$


where $\theta_{*}=\min _{\Omega} \theta_{0}$. By Lemma 3.5 we have

$$
\theta_{(n)} \geq I_{1} \geq \theta_{*}-c\left\|\theta_{(n)}-\theta_{0}\right\|_{W_{q, q_{0}}^{2,1}\left(\Omega^{t}\right)} t^{\sigma / 2}
$$

under the restriction $(6.13)$. From $(6.14)$ for $t$ so small that

$$
c\left\|\theta_{(n)}-\theta_{0}\right\|_{W_{q, q_{0}}^{2,1}\left(\Omega^{t}\right)} t^{\sigma / 2} \leq \theta_{*} / 2
$$

we have

$$
\theta_{(n)} \geq \theta_{*} / 2 .
$$

Now, we examine the expressions in the square brackets on the r.h.s. of (6.11). By the Hölder inequality the first term is bounded by

$$
\left\|\theta_{(n)} \varepsilon\left(u_{(n), t^{\prime}}\right)\right\|_{L_{q, q_{0}}\left(\Omega^{t}\right)} \leq\left\|\theta_{(n)}\right\|_{L_{\lambda_{1} q, \mu_{1} q_{0}}\left(\Omega^{t}\right)}\left\|\varepsilon\left(u_{(n), t^{\prime}}\right)\right\|_{L_{\lambda_{2} q, \mu_{2} q_{0}}\left(\Omega^{t}\right)} \equiv I_{2},
$$

where $1 / \lambda_{1}+1 / \lambda_{2}=1,1 / \mu_{1}+1 / \mu_{2}=1$. The first factor in $I_{2}$ is estimated by (see Lemma 3.1 )

$$
\begin{aligned}
\left\|\theta_{(n)}\right\|_{L_{\lambda_{1} q, \mu_{1} q_{0}}\left(\Omega^{t}\right)} & \leq \delta\left\|\theta_{(n)}\right\|_{W_{q, q_{0}}^{2}\left(\Omega^{t}\right)}+\varphi(1 / \delta)\left\|\theta_{(n)}\right\|_{L_{q, q_{0}}\left(\Omega^{t}\right)} \\
& \leq c_{5} t^{\alpha}\left(\left\|\theta_{(n)}\right\|_{W_{q, q_{0}}^{2,1}\left(\Omega^{t}\right)}+\left\|\theta_{0}\right\|_{L_{q}(\Omega)}\right)
\end{aligned}
$$

for some $\alpha>0$, under the restriction

$$
\frac{1}{q}+\frac{1}{q_{0}}-\frac{1}{\lambda_{1} q}-\frac{1}{\mu_{1} q_{0}}=\frac{1}{\lambda_{2}} \frac{1}{q}+\frac{1}{\mu_{2}} \frac{1}{q_{0}}<1,
$$

which holds in view of 6.12 .

The second factor in $I_{2}$ is bounded, in view of Lemma 3.1, by

$$
\begin{aligned}
\left\|\varepsilon\left(u_{(n), t^{\prime}}\right)\right\|_{L_{\lambda_{2} q, \mu_{2} q_{0}}\left(\Omega^{t}\right)} & \leq \delta\left\|u_{(n), t^{\prime}}\right\|_{W_{p, p_{0}}^{2,1}\left(\Omega^{t}\right)}+\varphi(1 / \delta)\left\|u_{(n), t^{\prime}}\right\|_{L_{q, q_{0}}\left(\Omega^{t}\right)} \\
& \leq c_{6} t^{\alpha}\left(\left\|u_{(n), t^{\prime}}\right\|_{W_{p, p_{0}}^{2,1}\left(\Omega^{t}\right)}+\left\|u_{1}\right\|_{L_{p}(\Omega)}\right)
\end{aligned}
$$

under the condition

$$
\frac{2}{p}+\frac{2}{p_{0}}-\frac{2}{\lambda_{2} q}-\frac{2}{\mu_{2} q_{0}}<2 .
$$

From (6.17), (6.18) and the relations between $\lambda_{i}, \mu_{i}, i=1,2$, we have

$$
\frac{1}{p}+\frac{1}{p_{0}}<2 .
$$

Moreover, the above estimates imply

$$
\begin{aligned}
I_{2} \leq & c_{7} t^{\alpha}\left(\left\|\theta_{(n)}\right\|_{W_{q, q_{0}}^{2,1}\left(\Omega^{t}\right)}+\left\|\theta_{0}\right\|_{L_{q}(\Omega)}\right) \\
& \times\left(\left\|u_{(n), t^{\prime}}\right\|_{W_{p, p_{0}}^{2,1}\left(\Omega^{t}\right)}+\left\|u_{1}\right\|_{L_{p}(\Omega)}\right) .
\end{aligned}
$$


The second term in the square brackets on the r.h.s. of 6.11 is bounded by (6.21)

$$
\begin{aligned}
\left\|\left|\nabla u_{(n), t^{\prime}}\right|^{2}\right\|_{L_{q, q_{0}}\left(\Omega^{t}\right)} & \leq\left\|\nabla u_{(n), t^{\prime}}\right\|_{L_{2 q, 2 q_{0}}\left(\Omega^{t}\right)}^{2} \\
& \leq\left(\delta\left\|u_{(n), t^{\prime}}\right\|_{W_{p, p_{0}}^{2,1}\left(\Omega^{t}\right)}+\varphi(1 / \delta)\left\|u_{(n), t^{\prime}}\right\|_{L_{p, p_{0}}\left(\Omega^{t}\right)}\right)^{2} \\
& \leq c_{8} t^{\alpha}\left(\left\|u_{(n), t^{\prime}}\right\|_{W_{p, p_{0}}^{2,1}\left(\Omega^{t}\right)}+\left\|u_{1}\right\|_{L_{p}(\Omega)}\right)^{2},
\end{aligned}
$$

under the restriction

$$
\frac{2}{p}+\frac{2}{p_{0}}-\frac{1}{q}-\frac{1}{q_{0}}<1
$$

In view of Lemma 3.1. From (6.8)-6.10) we have, for some $\alpha>0$,

$$
\left\|u_{(n+1), t^{\prime}}\right\|_{W_{p, p_{0}}^{2,1}\left(\Omega^{t}\right)} \leq \varphi\left(t^{\alpha} X_{n}(t), D(t)\right) .
$$

From 6.11, 6.20 and 6.21 we obtain

$$
\left\|\theta_{(n+1)}\right\|_{W_{q, q_{0}}^{2,1}\left(\Omega^{t}\right)} \leq \varphi\left(t^{\alpha} X_{n}(t), D(t)\right) .
$$

The above estimates hold under the restrictions 66.12, 6.19) and 6.22). Summarizing, inequalities (6.23) and (6.24) imply

$$
X_{n+1}(t) \leq \varphi_{1}\left(t^{\alpha} X_{n}(t), D(t)\right) .
$$

Hence, there exists a constant $\bar{A}$ such that for sufficiently small $t$,

$$
X_{0}(t) \leq \bar{A}, \quad \varphi_{1}\left(t^{\alpha} \bar{A}, D(t)\right) \leq \bar{A} .
$$

Then 6.26 implies

$$
X_{n}(t) \leq \bar{A} \quad \text { for any } n \in \mathbb{N}_{0} .
$$

This concludes the proof.

To show convergence of the sequence $\left\{u_{(n)}, \theta_{(n)}\right\}$ we introduce the differences

$$
U_{n}(t)=u_{(n)}(t)-u_{(n-1)}(t), \quad \vartheta_{n}(t)=\theta_{(n)}(t)-\theta_{(n-1)}(t),
$$

for $n \in \mathbb{N}$, which are solutions to the problems

$$
\begin{array}{ll}
U_{n+1, t t}-\nabla \cdot\left(A_{1} \varepsilon\left(U_{n+1, t}\right)\right)=\nabla \cdot\left(A_{2} \varepsilon\left(U_{n}\right)\right) & \\
\quad+\nabla \cdot\left(A\left(\theta_{(n)}-\theta_{(n-1)}\right)\right) & \text { in } \Omega^{T}, \\
U_{n+1}=0 & \text { on } S^{T}, \\
\left.U_{n+1}\right|_{t=0}=0,\left.\quad U_{n+1, t}\right|_{t=0}=0 & \text { in } \Omega,
\end{array}
$$


and

$$
\begin{array}{ll}
c_{v} \theta_{(n)}^{\sigma} \vartheta_{n+1, t}-\varkappa \Delta \vartheta_{n+1}=-c_{v}\left(\theta_{(n)}^{\sigma}-\theta_{(n-1)}^{\sigma}\right) \theta_{(n), t} & \\
\quad+\left(\theta_{(n)}-\theta_{(n-1)}\right) \varepsilon\left(u_{(n), t}\right)+\theta_{(n-1)} \varepsilon\left(U_{n, t}\right) & \\
\quad+A_{1} \varepsilon\left(U_{n, t}\right) \cdot \varepsilon\left(u_{(n), t}\right)+A_{1} \varepsilon\left(u_{(n-1), t}\right) \cdot \varepsilon\left(U_{n, t}\right) & \text { in } \Omega^{T}, \\
\bar{n} \cdot \nabla \vartheta_{n+1}=0 & \text { on } S^{T}, \\
\left.\vartheta_{n+1}\right|_{t=0}=0 & \text { in } \Omega .
\end{array}
$$

Let

$$
Y_{n}(t)=\left\|U_{n, t^{\prime}}\right\|_{W_{p^{\prime}, p_{0}^{\prime}}^{2,1}\left(\Omega^{t}\right)}+\left\|\vartheta_{n}\right\|_{W_{q^{\prime}, q_{0}^{\prime}}^{2,1}\left(\Omega^{t}\right)} .
$$

LEMMA 6.2. Let the assumptions of Lemma 6.1 hold. Then there exists a positive constant $d$ depending on $\bar{A}$ such that for some $\alpha>0$,

$$
Y_{n+1}(t) \leq d t^{\alpha} Y_{n}(t)
$$

Proof. Applying Lemma 3.6 to problem 6.29) yields

$$
\left\|U_{n+1, t^{\prime}}\right\|_{W_{p^{\prime}, p_{0}^{\prime}}^{2,1}\left(\Omega^{t}\right)} \leq c\left\|\nabla^{2} U_{n}\right\|_{L_{p^{\prime}, p_{0}^{\prime}}\left(\Omega^{t}\right)}+c\left\|\nabla \vartheta_{n}\right\|_{L_{p^{\prime}, p_{0}^{\prime}}\left(\Omega^{t}\right)} .
$$

By the formula

$$
U_{n}(t)=\int_{0}^{t} U_{n, t^{\prime}}\left(t^{\prime}\right) d t^{\prime},
$$

the first term on the r.h.s. of $(6.33)$ is estimated by

$$
t^{1 / p_{0}^{\prime}}\left\|U_{n, t^{\prime}}\right\|_{W_{p^{\prime}, p_{0}^{\prime}}^{2,1}\left(\Omega^{t}\right)} \text {. }
$$

We will also use the formula

$$
\vartheta_{n}(t)=\int_{0}^{t} \vartheta_{n, t^{\prime}}\left(t^{\prime}\right) d t^{\prime} .
$$

To estimate the second term on the r.h.s. of $(6.33)$ we express it in the form

$$
\left(\int_{0}^{t}\left\|\nabla \vartheta_{n}\left(t^{\prime}\right)\right\|_{L_{p^{\prime}}(\Omega)}^{p_{0}^{\prime}} d t^{\prime}\right)^{1 / p_{0}^{\prime}} \equiv I_{1} .
$$

Applying the interpolation

$$
\left\|\nabla \vartheta_{n}\right\|_{L_{p^{\prime}}(\Omega)} \leq c\left\|\vartheta_{n}\right\|_{W_{q^{\prime}}^{2}(\Omega)}^{\theta}\left\|\vartheta_{n}\right\|_{L_{q^{\prime}}(\Omega)}^{1-\theta}
$$

with $\theta=1 / q^{\prime}-1 / p^{\prime}+1 / 2$ yields

$$
\begin{aligned}
I_{1} & \leq c \sup _{t}\left\|\vartheta_{n}\right\|_{L_{q^{\prime}}(\Omega)}^{1-\theta}\left(\int_{0}^{t}\left\|\vartheta_{n}\left(t^{\prime}\right)\right\|_{W_{q^{\prime}}^{2}(\Omega)}^{p_{0}^{\prime} \theta} d t^{\prime}\right)^{1 / p_{0}^{\prime}} \\
& \leq c t^{\left(1-1 / q_{0}^{\prime}\right)(1-\theta)}\left\|\vartheta_{n, t^{\prime}}\right\|_{L_{q^{\prime}, q_{0}^{\prime}}^{1-\theta}\left(\Omega^{t}\right)}^{1-\vartheta_{n} \|_{L_{q_{0}^{\prime}}\left(0, t ; W_{q^{\prime}}^{2}(\Omega)\right)}^{\theta}},
\end{aligned}
$$


where for the second inequality we have used 6.35 and $p_{0}^{\prime} \theta \leq q_{0}^{\prime}$. Summarizing, we need the restriction

$$
p_{0}^{\prime}\left(1 / q^{\prime}-1 / p^{\prime}+1 / 2\right) \leq q_{0}^{\prime}, \quad 0<1 / q^{\prime}-1 / p^{\prime}+1 / 2<1 .
$$

Employing the above estimates in $(6.33)$ yields

$$
\left\|U_{n+1, t^{\prime}}\right\|_{W_{p^{\prime}, p_{0}^{\prime}}^{2,1}\left(\Omega^{t}\right)} \leq \varphi(\bar{A}) t^{\alpha} Y_{n}(t) .
$$

Applying Lemma 3.7 to 6.30 gives

$$
\begin{aligned}
\left\|\vartheta_{n+1}\right\|_{W_{q^{\prime}, q_{0}^{\prime}}^{2,1}\left(\Omega^{t}\right)} \leq & \varphi(\bar{A})\left[\left\|\vartheta_{n} \theta_{(n), t^{\prime}}\right\|_{L_{q^{\prime}, q_{0}^{\prime}}\left(\Omega^{t}\right)}+\left\|\vartheta_{n} \varepsilon\left(u_{(n), t^{\prime}}\right)\right\|_{L_{q^{\prime}, q_{0}^{\prime}}\left(\Omega^{t}\right)}\right. \\
& \left.+\left\|\varepsilon\left(U_{n, t^{\prime}}\right)\right\|_{L_{q^{\prime}, q_{0}^{\prime}}} \Omega^{t}\right) \\
& +\left\|\varepsilon\left(U_{n, t^{\prime}}\right) \varepsilon\left(u_{(n), t^{\prime}}\right)\right\|_{L_{q^{\prime}, q_{0}^{\prime}}\left(\Omega^{t}\right)} \\
& \left.+\left\|\varepsilon\left(u_{(n-1), t^{\prime}}\right) \varepsilon\left(U_{n, t^{\prime}}\right)\right\|_{L_{q^{\prime}, q_{0}^{\prime}}\left(\Omega^{t}\right)}\right] .
\end{aligned}
$$

Now, we examine the particular terms from the r.h.s. of (6.38). The first term in the square brackets is treated as follows:

$$
\begin{aligned}
\left\|\vartheta_{n} \theta_{(n), t^{\prime}}\right\|_{L_{q^{\prime}, q_{0}^{\prime}}\left(\Omega^{t}\right)} & =\left(\int_{0}^{t}\left\|\vartheta_{n} \theta_{(n), t^{\prime}}\right\|_{L_{q^{\prime}}(\Omega)}^{q_{0}^{\prime}} d t^{\prime}\right)^{1 / q_{0}^{\prime}} \\
& \leq\left(\int_{0}^{t}\left\|\vartheta_{n}\right\|_{L_{\lambda_{1} q^{\prime}}(\Omega)}^{q_{0}^{\prime}}\left\|\theta_{(n), t^{\prime}}\right\|_{L_{\lambda_{2} q^{\prime}}(\Omega)}^{q_{0}^{\prime}} d t^{\prime}\right)^{1 / q_{0}^{\prime}} \equiv J_{1},
\end{aligned}
$$

where $1 / \lambda_{1}+1 / \lambda_{2}=1$. Setting $\lambda_{2} q^{\prime}=q$, we have $\lambda_{2}=q / q^{\prime}$ so $\lambda_{1}=$ $q /\left(q-q^{\prime}\right)$. Then

$$
J_{1} \leq\left(\int_{0}^{t}\left\|\vartheta_{n}\right\|_{L_{\frac{q q^{\prime}}{q-q^{\prime}}}^{q^{\prime}}}^{q_{0}}\left\|\theta_{(n), t^{\prime}}\right\|_{L_{q}(\Omega)}^{q_{0}^{\prime}} d t^{\prime}\right)^{1 / q_{0}^{\prime}} \equiv J_{1}^{1} .
$$

We use the interpolation (see Lemma 3.3.

$$
\left\|\vartheta_{n}\right\|_{L_{\frac{q q^{\prime}}{q-q^{\prime}}}(\Omega)} \leq c\left\|\vartheta_{n}\right\|_{W_{q^{\prime}}^{2}(\Omega)}^{\theta}\left\|\vartheta_{n}\right\|_{L_{q^{\prime}}(\Omega)}^{1-\theta},
$$

where $\theta$ satisfies the equation

$$
\frac{2}{q q^{\prime} /\left(q-q^{\prime}\right)}=(1-\theta) \frac{2}{q^{\prime}}+\theta\left(\frac{2}{q^{\prime}}-2\right) .
$$

Solving the equation yields $\theta=1 / q$. Then

$$
J_{1}^{1} \leq c \sup _{t}\left\|\vartheta_{n}\right\|_{L_{q^{\prime}}(\Omega)}^{1-1 / q}\left(\int_{0}^{t}\left\|\vartheta_{n}\right\|_{W_{q^{\prime}}^{2}(\Omega)}^{q_{0}^{\prime} / q}\left\|\theta_{(n), t^{\prime}}\right\|_{L_{q}(\Omega)}^{q_{0}^{\prime}} d t^{\prime}\right)^{1 / q_{0}^{\prime}} \equiv J_{1}^{2} .
$$


Applying (6.35) and the Hölder inequality to the integrand yields

$$
\begin{aligned}
J_{1}^{2} \leq & c t^{\left(1-1 / q_{0}^{\prime}\right)(1-1 / q)}\left\|\vartheta_{n, t^{\prime}}\right\|_{L_{q^{\prime}, q_{0}^{\prime}}^{1-1 / q}\left(\Omega^{t}\right)}^{t}\left(\int_{0}^{t}\left\|\vartheta_{n}\right\|_{W_{q^{\prime}}^{2}(\Omega)}^{q_{0}^{\prime} \lambda_{1} / q} d t^{\prime}\right)^{1 /\left(q_{0}^{\prime} \lambda_{1}\right)} \\
& \times\left(\int_{0}^{t}\left\|\theta_{(n), t^{\prime}}\right\|_{L_{q}(\Omega)}^{q_{0}^{\prime} \lambda_{2}} d t^{\prime}\right)^{1 /\left(q_{0}^{\prime} \lambda_{2}\right)} \equiv J_{1}^{3},
\end{aligned}
$$

where $1 / \lambda_{1}+1 / \lambda_{2}=1$. Setting $\lambda_{1}=q$, we have $\lambda_{2}=q /(q-1)$. Next, we need $q_{0}^{\prime} q /(q-1)=q_{0}$, so $q_{0}^{\prime}=q_{0}(1-1 / q)$. Then

$$
J_{1}^{3} \leq t^{1-1 / q-1 / q_{0}}\left\|\vartheta_{n, t^{\prime}}\right\|_{L_{q^{\prime}, q_{0}^{\prime}}^{(-1 / q}}^{\left.1-\Omega^{t}\right)}\left\|\vartheta_{n}\right\|_{L_{q_{0}^{\prime}}\left(0, t ; W_{q^{\prime}}^{2}(\Omega)\right)}^{1 / q}\left\|\theta_{(n), t^{\prime}}\right\|_{L_{q, q_{0}}\left(\Omega^{t}\right)} .
$$

Hence, in view of (6.7) we have

$$
\left\|\vartheta_{n} \theta_{(n), t^{\prime}}\right\|_{L_{q^{\prime}, q_{0}^{\prime}}\left(\Omega^{t}\right)} \leq t^{1-1 / q-1 / q_{0}} \bar{A}\left\|\vartheta_{n}\right\|_{W_{q^{\prime}, q_{0}^{\prime}}^{2,1}\left(\Omega^{t}\right)},
$$

where

$$
q_{0}^{\prime}=q_{0}(1-1 / q)
$$

The second term in the square brackets on the r.h.s. of (6.38) is bounded by

$$
\left(\int_{0}^{t}\left\|\vartheta_{n}\right\|_{L_{\lambda_{1} q^{\prime}}(\Omega)}^{q^{\prime}}\left\|\varepsilon\left(u_{(n), t^{\prime}}\right)\right\|_{L_{\lambda_{2} q^{\prime}}(\Omega)}^{q_{0}^{\prime}} d t^{\prime}\right)^{1 / q_{0}^{\prime}} \equiv J_{2},
$$

where $1 / \lambda_{1}+1 / \lambda_{2}=1$. Applying Lemmas 3.2 and 3.3 we get

$$
J_{2} \leq \sup _{t}\left\|\vartheta_{n}\right\|_{L_{q^{\prime}}(\Omega)}^{1-\theta}\left(\int_{0}^{t}\left\|\vartheta_{n}\right\|_{W_{q^{\prime}}^{2}(\Omega)}^{\theta q^{\prime}}\left\|u_{(n), t^{\prime}}\right\|_{W_{p}^{2}(\Omega)}^{q_{0}^{\prime}} d t^{\prime}\right)^{1 / q_{0}^{\prime}} \equiv J_{2}^{1},
$$

where $2 / p-2 /\left(\lambda_{2} q^{\prime}\right) \leq 1$ and $\frac{2}{\lambda_{1} q^{\prime}}=(1-\theta) \frac{2}{q^{\prime}}+\theta\left(\frac{2}{q^{\prime}}-2\right)$ so $\theta=1 /\left(\lambda_{2} q^{\prime}\right)$. Hence $2 / p-2 \theta \leq 1$.

Using 6.35 and applying the Hölder inequality to the time integral yields

$$
\begin{aligned}
J_{2}^{1} \leq & t^{\left(1-1 / q_{0}^{\prime}\right)(1-\theta)}\left\|\vartheta_{n, t^{\prime}}\right\|_{L_{q^{\prime}, q_{0}^{\prime}}^{(-\theta}}^{1-\theta}\left(\int_{0}^{t}\left\|\vartheta_{n}\right\|_{W_{q^{\prime}}^{2}(\Omega)}^{\theta \mu_{1} q_{0}^{\prime}} d t^{\prime}\right)^{1 /\left(\mu_{1} q_{0}^{\prime}\right)} \\
& \times\left(\int_{0}^{t}\left\|u_{(n), t^{\prime}}\right\|_{W_{p}^{2}(\Omega)}^{q_{0}^{\prime} \mu_{2}} d t^{\prime}\right)^{1 /\left(q_{0}^{\prime} \mu_{2}\right)} \equiv J_{2}^{3}
\end{aligned}
$$

where $1 / \mu_{1}+1 / \mu_{2}=1$. Setting $\theta \mu_{1} q_{0}^{\prime}=q_{0}^{\prime}$ and $q_{0}^{\prime} \mu_{2}=p_{0}$ we get $\mu_{2}=p_{0} / q_{0}^{\prime}$, $\theta=1 / \mu_{1}=1-q_{0}^{\prime} / p_{0}$. But $\theta$ satisfies $1 / p-\theta \leq 1 / 2$. Hence

$$
\left\|\vartheta_{n} \varepsilon\left(u_{(n), t^{\prime}}\right)\right\|_{L_{q^{\prime}, q_{0}^{\prime}}\left(\Omega^{t}\right)} \leq t^{\left(q_{0}^{\prime}-1\right) / p_{0}} \bar{A}\left\|\vartheta_{n}\right\|_{W_{q^{\prime}, q_{0}^{\prime}}^{2,1}\left(\Omega^{t}\right)}
$$

under the restrictions

$$
1 / p+q_{0}^{\prime} / p_{0} \leq 3 / 2, \quad q_{0}^{\prime} / p_{0}<1, \quad 1 / p-1 / 2<1 / q^{\prime} .
$$


The third term in the square brackets in 6.38 is bounded by (see Lemmas 3.2 and 3.3

$$
\begin{aligned}
\left\|\varepsilon\left(U_{n, t}\right)\right\|_{L_{q^{\prime}, q_{0}^{\prime}}\left(\Omega^{t}\right)} & \leq\left(\int_{0}^{t}\left\|U_{n, t^{\prime}}\right\|_{W_{q^{\prime}}^{1}(\Omega)}^{q_{0}^{\prime}} d t^{\prime}\right)^{1 / q_{0}^{\prime}} \\
& \leq c\left(\int_{0}^{t}\left\|U_{n, t^{\prime}}\right\|_{W_{p^{\prime}}^{2}(\Omega)}^{q_{0}^{\prime} \theta}\left\|U_{n, t^{\prime}}\right\|_{L_{p^{\prime}}(\Omega)}^{q^{\prime}(1-\theta)} d t^{\prime}\right)^{1 / q_{0}^{\prime}} \equiv J_{3},
\end{aligned}
$$

where $\theta=1 / p^{\prime}+1 / 2-1 / q^{\prime}$ and $0<\theta<1$. Continuing, we have

$$
J_{3} \leq c \sup _{t}\left\|U_{n, t}\right\|_{L_{p^{\prime}}(\Omega)}^{1-\theta}\left(\int_{0}^{t}\left\|U_{n, t^{\prime}}\right\|_{W_{p^{\prime}}^{2}(\Omega)}^{q_{0}^{\prime} \theta} d t^{\prime}\right)^{1 / q_{0}^{\prime}} \equiv J_{3}^{1} .
$$

Using the formula

$$
U_{n, t}=\int_{0}^{t} U_{n, t^{\prime} t^{\prime}} d t^{\prime}
$$

and setting $q_{0}^{\prime} \theta \leq p_{0}^{\prime}$ we obtain

$$
J_{3}^{1} \leq c t^{\left(1-1 / p_{0}^{\prime}\right)(1-\theta)}\left\|U_{n, t^{\prime}}\right\|_{W_{p^{\prime}, p_{0}^{\prime}}^{2,1}\left(\Omega^{t}\right)},
$$

where

$$
q_{0}^{\prime}\left(\frac{1}{p^{\prime}}+\frac{1}{2}-\frac{1}{q^{\prime}}\right) \leq p_{0}^{\prime}
$$

The last two terms in the square brackets on the r.h.s. of 6.38 can be estimated in the same way. Therefore, it remains to examine the last but one term:

$$
\begin{aligned}
& \left\|\varepsilon\left(U_{n, t}\right) \varepsilon\left(u_{(n), t}\right)\right\|_{L_{q^{\prime}, q_{0}^{\prime}}\left(\Omega^{t}\right)} \\
& \leq\left(\int_{0}^{t}\left\|\varepsilon\left(U_{n, t^{\prime}}\right)\right\|_{L_{\lambda_{1} q^{\prime}}(\Omega)}^{q^{\prime}}\left\|\varepsilon\left(u_{(n), t^{\prime}}\right)\right\|_{L_{\lambda_{2} q^{\prime}}(\Omega)}^{q^{\prime}} d t^{\prime}\right)^{1 / q_{0}^{\prime}} \equiv J_{4},
\end{aligned}
$$

where $1 / \lambda_{1}+1 / \lambda_{2}=1$. By Lemmas 3.2 and 3.3 we have

$$
J_{4} \leq c\left(\int_{0}^{t}\left\|U_{n, t^{\prime}}\right\|_{W_{p^{\prime}}^{2}(\Omega)}^{\theta q^{\prime}}\left\|U_{n, t^{\prime}}\right\|_{L_{p^{\prime}}(\Omega)}^{(1-\theta) q_{0}^{\prime}}\left\|u_{(n), t^{\prime}}\right\|_{W_{p}^{2}(\Omega)}^{q_{0}^{\prime}} d t^{\prime}\right)^{1 / q_{0}^{\prime}} \equiv J_{4}^{1},
$$

where $\frac{2}{\lambda_{1} q^{\prime}}-1=(1-\theta) \frac{2}{p^{\prime}}+\theta\left(\frac{2}{p^{\prime}}-2\right)$ so $\theta=\frac{1}{p^{\prime}}+\frac{1}{2}-\frac{1}{\lambda_{1} q^{\prime}}, 0<\theta<1$ and $\frac{2}{p}-\frac{2}{\lambda_{2} q^{\prime}} \leq 1$. In view of 6.41 we have

$$
\begin{aligned}
J_{4}^{1} \leq & c t^{\left(1-1 / p_{0}^{\prime}\right)(1-\theta)}\left\|U_{n, t^{\prime} t^{\prime}}\right\|_{L_{p^{\prime}, p_{0}^{\prime}}^{1-\theta}\left(\Omega^{t}\right)}^{1-\theta} \\
& \times\left(\int_{0}^{t}\left\|U_{n, t^{\prime}}\right\|_{W_{p^{\prime}}^{2}(\Omega)}^{\theta q_{0}^{\prime}}\left\|u_{(n), t^{\prime}}\right\|_{W_{p}^{2}(\Omega)}^{q_{0}^{\prime}} d t^{\prime}\right)^{1 / q_{0}^{\prime}} \equiv J_{4}^{2} .
\end{aligned}
$$


Applying the Hölder inequality to the time integral yields

$$
\begin{aligned}
J_{4}^{2} \leq & c t^{\left(1-1 / p_{0}^{\prime}\right)(1-\theta)}\left\|U_{n, t^{\prime} t^{\prime}}\right\|_{L_{p^{\prime}, p_{0}^{\prime}}^{1}\left(\Omega^{t}\right)}^{1-\theta}\left(\int_{0}^{t}\left\|U_{n, t^{\prime}}\right\|_{W_{p^{\prime}}^{2}(\Omega)}^{\theta q_{0}^{\prime} \mu_{1}} d t^{\prime}\right)^{1 /\left(q_{0}^{\prime} \mu_{1}\right)} \\
& \times\left(\int_{0}^{t}\left\|u_{(n), t^{\prime}}\right\|_{W_{p}^{2}(\Omega)}^{q_{0}^{\prime} \mu_{2}} d t^{\prime}\right)^{1 /\left(q_{0}^{\prime} \mu_{2}\right)} \equiv J_{4}^{3}
\end{aligned}
$$

where $1 / \mu_{1}+1 / \mu_{2}=1$. Setting $\theta q_{0}^{\prime} \mu_{1} \leq p_{0}^{\prime}$ and $q_{0}^{\prime} \mu_{2} \leq p_{0}$ we obtain

$$
J_{4}^{3} \leq c \bar{A} t^{\left(1-1 / p_{0}^{\prime}\right)(1-\theta)}\left\|U_{n, t^{\prime}}\right\|_{W_{p^{\prime}, p_{0}^{\prime}}^{2,1}\left(\Omega^{t}\right)},
$$

where

$$
\frac{q_{0}^{\prime}}{p_{0}^{\prime}}\left(\frac{1}{p}+\frac{1}{p^{\prime}}-\frac{1}{q^{\prime}}\right) \leq 1 .
$$

the restrictions (6.36), 6.38), 6.40, 6.42, 6.43) are satisfied if

$$
\left|\frac{1}{q^{\prime}}-\frac{1}{p^{\prime}}\right|<\frac{1}{2}, \quad \frac{q_{0}^{\prime}}{p_{0}^{\prime}} \leq 1, \quad \frac{1}{p}<\frac{1}{2}, \quad \frac{q_{0}^{\prime}}{p_{0}} \leq 1,
$$

$q_{0}^{\prime}=q_{0}-1<p_{0}$. Hence the lemma is proved.

TheOREm 6.3 (local existence). Let the assumptions of Lemma 6.1 hold. Then for a sufficiently small time $T$ there exists a solution to problem (1.1)(1.6) such that there exists a constant $\bar{A}$ depending on $D$ and $T$ and

$$
X(t)=\left\|u_{, t^{\prime}}\right\|_{W_{p, p_{0}}^{2,1}\left(\Omega^{t}\right)}+\|\theta\|_{W_{q, q_{0}}^{2,1}\left(\Omega^{t}\right)} \leq \bar{A}
$$

for all $t \leq T$.

7. Proof of the Main Theorem. To prove global existence of solutions to problem (1.1)-1.4 we need the existence of local solutions showed in Section 6 and the global a priori estimates stated in Section 5 . Then global existence is proved step by step in time. In Section 6 we proved local existence of solutions such that

$$
\begin{aligned}
& u_{, t} \in W_{p, p_{0}}^{2,1}\left(\Omega^{T}\right), \quad \theta \in W_{q, q_{0}}^{2,1}\left(\Omega^{T}\right), \\
& \frac{1}{p}+\frac{1}{p_{0}}<1, \quad \frac{1}{q}+\frac{1}{q_{0}}<1, \quad q_{0}>2 .
\end{aligned}
$$

To prove (7.1) we need the following regularity of data:

$$
\begin{aligned}
& b \in L_{p, p_{0}}\left(\Omega^{T}\right), \quad u_{0} \in W_{p}^{2}(\Omega), \quad u_{1} \in B_{p, p_{0}}^{2-2 / p_{0}}(\Omega), \\
& \theta_{0} \in B_{q, q_{0}}^{2-2 / q_{0}}(\Omega), \quad g \in L_{q, q_{0}}\left(\Omega^{t}\right) .
\end{aligned}
$$


To show global estimates in Section 5 we need (see Lemmas 5.5, 5.6, 5.8, Corollary 5.9

$$
\begin{aligned}
& g \in L_{1}\left(0, T ; L_{\infty}(\Omega)\right) \cap L_{2}\left(0, T ; L_{4}(\Omega)\right), \quad b \in L_{4}\left(\Omega^{t}\right), \\
& u_{1} \in B_{4,4}^{3 / 2}(\Omega), \quad u_{0} \in W_{4}^{2}(\Omega), \quad \theta_{0} \in C^{\alpha}(\Omega), \quad \alpha>0, \quad \theta \geq \theta_{*}>0 .
\end{aligned}
$$

In view of the imbedding

$$
\left\|\theta_{0}\right\|_{C^{\alpha}(\Omega)} \leq c\left\|\theta_{0}\right\|_{B_{q, q_{0}}^{2-2 / q_{0}}(\Omega)}, \quad \frac{2}{q}+\frac{2}{q_{0}}+\alpha<1,
$$

we see that (7.2) and (7.3) are compatible if

$$
g \in L_{q, q_{0}}\left(\Omega^{T}\right) \cap L_{1}\left(0, T ; L_{\infty}(\Omega)\right) .
$$

This concludes the proof.

Uniqueness can be proved in the standard way.

Acknowledgments. The authors thank the referee for important and valuable comments.

\section{References}

[BIN] O. V. Besov, V. P. Il'in and S. M. Nikol'skiř, Integral Representation of Functions and Imbedding Theorems, Nauka, Moscow, 1975 (in Russian).

[BG] D. Blanchard and O. Guibé, Existence of a solution for a nonlinear system in thermoviscoelasticity, Adv. Differential Equations 5 (2000), 1221-1252.

[B] Ya. S. Bugrov, Function spaces with mixed norm, Math. USSR-Izv. 5 (1971), 1145-1167.

[C] B. D. Coleman, Thermodynamics of materials with memory, Arch. Ration. Mech. Anal. 17 (1964), 1-46.

[D] C. M. Dafermos, Global smooth solutions to the initial-boundary value problem for the equations of one-dimensional nonlinear thermoviscoelasticity, SIAM J. Math. Anal. 13 (1982), 397-408.

[DH] C. M. Dafermos and L. Hsiao, Global smooth thermomechanical processes in onedimensional nonlinear thermoviscoelasticity, Nonlinear Anal. 6 (1982), 435-454.

[EJK] D. Eck, J. Jarušek and M. Krbec, Unilateral Contact Problems: Variational Methods and Existence Theorems, Chapman \& Hall/CRC, Boca Raton, FL, 2005.

[GA] J. A. Gawinecki, Global existence of solutions for non-small data to non-linear spherically symmetric thermoviscoelasticity, Math. Methods Appl. Sci. 26 (2003), 907-936.

[GZ1] J. A. Gawinecki and W. M. Zajączkowski, Global non-small data existence of spherically symmetric solutions to nonlinear viscoelasticity in a ball, Z. Anal. Anwend. 30 (2011), 387-419.

[GZ2] J. A. Gawinecki and W. M. Zajączkowski, On global existence of solutions of the Neumann problem for spherically symmetric nonlinear viscoelasticity in a ball, ISRN Math. Anal. 2013, art. ID 268505, 13 pp.

[GZ3] J. A. Gawinecki and W. M. Zajączkowski, Global existence of solutions to the nonlinear thermoviscoelasticity system with small data, Topol. Methods Nonlinear Anal. 39 (2012), 263-284. 
[GZ4] J. A. Gawinecki and W. M. Zajączkowski, Global regular solutions to two-dimensional thermoviscoelasticity, Comm. Pure Appl. Anal. 15 (2016), 1009-1028.

[G] K. K. Golovkin, On equivalent norms for fractional spaces, Amer. Math. Soc. Transl. (2) 81 (1969), 257-280.

[K] N. V. Krylov, The Calderón-Zygmund theorem and its application for parabolic equations, Algebra i Analiz 13 (2001), 1-25 (in Russian).

[LSU] O. A. Ladyzhenskaya, V. A. Solonnikov and N. N. Ural'tseva, Linear and Quasilinear Equations of Parabolic Type, Nauka, Moscow, 1967 (in Russian).

[LM] J.-L. Lions et E. Magenes, Problèmes aux limites non homogènes et applications, Vol. 1, Dunod, Paris, 1968.

[PZ1] I. Pawłow and W. M. Zajączkowski, Global regular solutions to a Kelvin-Voigt type thermoviscoelastic system, SIAM J. Math. Anal. (2013), 1997-2045.

[PZ2] I. Pawłow and W. M. Zajączkowski, Unique solvability of a nonlinear thermoviscoelasticity system in Sobolev space with a mixed norm, Discrete Contin. Dynam. Systems Ser. S 4 (2011), 441-466.

[RR] R. Rossi and T. Roubíček, Thermodynamics and analysis of rate-independent adhesive contact at small strains, Nonlinear Anal. 74 (2011), 3159-3190.

[R1] T. Roubíček, Thermo-visco-elasticity at small strains with $L^{1}$-data, Quart. Appl. Math. 67 (2009), 47-71.

[R2] T. Roubíček, Thermodynamics of rate-independent processes in viscous solids at small strains, SIAM J. Math. Anal. 42 (2010), 256-297.

[R3] T. Roubíček, Modelling of thermodynamics of martensitic transformation in shape memory alloys, Discrete Contin. Dynam. Systems Suppl. 2007, 892-902.

[SL] M. Slemrod, Global existence, uniqueness and asymptotic stability of classical smooth solutions in one-dimensional non-linear thermoviscoelasticity, Arch. Ration. Mech. Anal. 76 (1981), 97-133.

[S1] V. A. Solonnikov, Estimates of solutions of the Stokes equations in S. L. Sobolev spaces with a mixed norm, Zap. Nauchn. Sem. S.-Peterburg. Otdel. Mat. Inst. Steklova (POMI) 288 (2002), 204-231 (in Russian).

[S2] V. A. Solonnikov, On boundary value problems for linear parabolic systems of differential equations of general type, Trudy Mat. Inst. Steklova 83 (1965) (in Russian).

Jerzy A. Gawinecki

Institute of Mathematics and Cryptology

Cybernetics Faculty

Military University of Technology

S. Kaliskiego 2

00-908 Warszawa, Poland

E-mail: jgawinecki@wat.edu.pl
Wojciech M. Zajączkowski Institute of Mathematics Polish Academy of Sciences Śniadeckich 8 00-656 Warszawa, Poland and Institute of Mathematics and Cryptology Cybernetics Faculty Military University of Technology S. Kaliskiego 2 00-908 Warszawa, Poland E-mail:wz@impan.pl 
